\title{
INTEGRABILITY OF EXPECTED INCREMENTS OF POINT PROCESSES AND A RELATED RANDOM CHANGE OF SCALE
}

\author{
BY \\ F. PAPANGELOU( $\left.{ }^{(}\right)$
}

\begin{abstract}
Given a stationary point process with finite intensity on the real line $R$, denote by $N(Q)(Q$ Borel set in $R)$ the random number of points that the process throws in $Q$ and by $\mathscr{F}_{t}(t \in R)$ the $\sigma$-field of events that happen in $(-\infty, t)$. The main results are the following. If for each partition $\Delta=\left\{b=\xi_{0}<\xi_{1}<\cdots<\xi_{n+1}=c\right\}$ of an interval $[b, c]$ we set $S_{\Delta}(\omega)=\sum_{v=0}^{n} E\left(N\left[\xi_{v}, \xi_{v+1}\right) \mid \mathscr{F}_{\xi_{v}}\right)$ then $\lim _{\Delta} S_{\Delta}(\omega)=W(\omega,[b, c))$ exists a.s. and in the mean when $\max _{0 \leqq v \leqq n}\left(\xi_{v+1}-\xi_{v}\right) \rightarrow 0$ (the a.s. convergence requires a judicious choice of versions). If the random transformation $t \Rightarrow W(\omega,[0, t))$ of $[0, \infty)$ onto itself is a.s. continuous (i.e. without jumps), then it transforms the nonnegative points of the process into a Poisson process with rate 1 and independent of $\mathscr{F}_{0}$. The ratio $\varepsilon^{-1} E\left(N[0, \varepsilon) \mid \mathscr{F}_{0}\right)$ converges a.s. as $\varepsilon \downarrow 0$. A necessary and sufficient condition for its convergence in the mean (as well as for the a.s. absolute continuity of the function $W[0, t)$ on $(0, \infty))$ is the absolute continuity of the Palm conditional probability $P_{0}$ relative to the absolute probability $P$ on the $\sigma$-field $\mathscr{F}_{0}$. Further results are described in $\S 1$.
\end{abstract}

1. Introduction. The present paper, dealing with (mostly stationary) point processes on the real line, is a natural sequel to [5], where many of the results were obtained in partial form (more details are given at the end of this introduction). The processes studied here are assumed to have finite intensities and no multiple points.

For a quick description of the main results let the random variable $N(Q)$ denote the number of points of the process in the Borel set $Q$ and for each real $t$ let $\mathscr{F}_{t}$ be the $\sigma$-field of events that happen left of $t$, i.e. the $\sigma$-field generated by the random variables $N(Q)$ with $Q \subseteq(-\infty, t)$. Given an interval $[b, c]$ and a partition $\Delta=\left\{b=\xi_{0}<\xi_{1}<\cdots<\xi_{n+1}=c\right\}$ of $[b, c]$ we set

$$
S_{\Delta}(\omega)=\sum_{\nu=0}^{n} E\left(N\left[\xi_{v}, \xi_{v+1}\right) \mid \mathscr{F}_{\xi_{v}}\right) .
$$

In $\S 3$ and $\S 4$ we study the behaviour of the random sums $S_{\Delta}$ when the mesh $\|\Delta\|=\max _{1 \leqq v \leqq n}\left(\xi_{v+1}-\xi_{v}\right)$ goes to zero. We show (Theorem 1) that, with the right

Received by the editors March 1, 1971.

AMS 1969 subject classifications. Primary 6050, 6069; Secondary 2645, 2646.

Key words and phrases. Stochastic point process, Poisson process, Palm probability, Burkill integrability, conditional expectation, almost sure convergence, mean convergence, absolute continuity.

(1) Supported by NSF grant GP-21339. 
choice of versions, these sums converge almost surely to a random variable $W(\omega,[b, c))$ and that they also converge in the mean (Theorem 3). A consequence of the a.s. convergence is the existence of $\lim _{\varepsilon \downarrow 0} \varepsilon^{-1} E\left(N[0, \varepsilon) \mid \mathscr{F}_{0}\right)$ a.s. (using the "correct" versions). The latter, however, is not always a mean limit and a necessary and sufficient condition for this is described below.

In $\S 5$ we prove that if the random function $\varphi_{\omega}(t)=W(\omega,[0, t))(t>0)$ is a.s. continuous and if $t_{1}(\omega)<t_{2}(\omega)<\cdots$ are the nonnegative points of the process then the process $\tau_{1}(\omega) \leqq \tau_{2}(\omega) \leqq \cdots$, where $\tau_{n}(\omega)=W\left(\omega,\left[0, t_{n}(\omega)\right)\right)$, is a Poisson process in $[0, \infty)$ with rate 1 and independent of $\mathscr{F}_{0}$ (Theorem 6$)$. The proof of this is based on a characterization of the Poisson process (Theorem 5) proved in [5]. Combining this with the fact that $W$ is the limit of sums such as (1) we obtain the limit Theorem 7. The "physical" interpretation of Theorems 6 and 7 is given in the text: Roughly, moving in $[0, \infty)$ so as to meet expected future points at a rate of one per time unit (given at each instant complete knowledge of the past), we meet them at the times of a Poisson process. In other words, under the assumption made, by smoothing the "conditional rate" of the process we make it into a Poisson process.

Let now $P_{0}$ be the Palm probability of the process, i.e. the conditional probability measure given that the process has a point at 0 . If $P$ denotes the absolute probability measure then the following conditions are equivalent:

(i) $P_{0} \ll P$ on $\mathscr{F}_{0}$ ( $\ll$ means absolute continuity);

(ii) $\lim _{\varepsilon \downarrow 0} \varepsilon^{-1} E\left(N[0, \varepsilon) \mid \mathscr{F}_{0}\right)$ exists in the mean;

(iii) the function $\varphi_{\omega}(t)=W(\omega,[0, t))$ is a.s. absolutely continuous relative to the Lebesgue measure.

In this case if we denote by $X$ the Radon-Nikodym derivative whose existence is implied by (i), and by $X(t),-\infty<t<\infty$, the translates of $X$, then $W(\omega,[b, c))$ $=\alpha \int_{b}^{c} X(t, \omega) d t$. These statements are enunciated in Theorems 9 and 10. Theorem 11 gives a criterion for the condition " $P_{0} \ll P$ on $\mathscr{F}_{0}$ ", which is expressed in terms of $P_{0}$ alone. Using this criterion we prove in Theorem 12 that if $P_{0} \ll P$ on $\mathscr{F}_{-\varepsilon}$ for every $\varepsilon>0$ then $P_{0} \ll P$ on $\mathscr{F}_{0}$. Finally, Theorem 13 is a sharpened version of a theorem in [5] and Theorems 8 and 14 state that $W$ is a.s. continuous or absolutely continuous if and only if the conditional distribution (under $P_{0}$ ) of the first positive point, given $\mathscr{F}_{0}$, is a.s. continuous or absolutely continuous respectively.

Of the main results, Theorems 3 and 6 are proved in [5] for the case where $P_{0} \ll P$ on $\mathscr{F}_{0}$. Theorem 3 (the mean convergence of $S_{\Delta}$ ) now dispenses with all restrictive assumptions (except stationariness) and its present proof is based on the (new) a.s. convergence Theorem 1. Theorem 6 relaxes the assumption to one which is optimal, but the proof is only an adaptation of that in [5]. We give most of this proof here (except for some technical propositions) for two reasons, first in order to indicate the changes (use of the Radon-Nikodym derivative is now replaced by two applications of Theorem 4) and second because this is one of the basic results. The sufficiency of the condition " $P_{0} \ll P$ on $\mathscr{F}_{0}$ " in Theorems 9 and 10 (but not the 
necessity) is already proved in [5], as is a weak-convergence version of Theorem 7, which is now established as an a.s.-convergence theorem.

After the above results were obtained I learned from $\mathrm{H}$. Rost that sums of conditional increments such as those in (1) had been used by K. Murali Rao [4] in a new proof of Meyer's decomposition theorem for continuous parameter supermartingales. His convergence results are naturally weaker than ours, since his processes are more general. We make some comments on the connection between his work and ours in [6].

The Burkill integral has of course proved useful in the study of other aspects of point processes. It has been employed for example by F. Zítek (see [9]) and by W. Fieger in a series of papers, the latest of which has appeared recently [2]. However, Murali Rao's is the only other work I am aware of in which it has been used with conditioning.

I am indebted to H. Rost for many invaluable discussions and to the referee for his many suggestions which have resulted in the elimination of some obscurities.

2. Groundwork. Let $R$ be the real line and $\mathscr{B}$ the $\sigma$-field of Borel subsets of $R$. Following [8] we let $\Omega$ be the space of all countable subsets of $R$ which are unbounded on both left and right and are locally finite (i.e. have no finite accumulation point). Such sets will be denoted by $\omega$. If $\omega \in \Omega$ and $Q \in \mathscr{B}$, we denote by $N(\omega, Q)$ the number of points of $\omega$ in $Q$ and we let $\mathscr{F}$ be the minimal $\sigma$-field relative to which all functions $N(\omega, Q)(Q$ bounded Borel set in $R$ ) are measurable. A point process in $R$ is defined by a probability measure $P$ on $\mathscr{F}$.

If $Q \subseteq R, A \in \mathscr{F}$ and $t \in R$, we set $Q+t=\{x+t: x \in Q\}, A+t=\{\omega+t: \omega \in A\}$. A point process is said to be stationary if

$$
P\left(N\left(Q_{1}\right)=j_{1}, \ldots, N\left(Q_{n}\right)=j_{n}\right)=P\left(N\left(Q_{1}+t\right)=j_{1}, \ldots, N\left(Q_{n}+t\right)=j_{n}\right)
$$

for any bounded Borel sets $Q_{1}, \ldots, Q_{n}$, any nonnegative integers $j_{1}, \ldots, j_{n}$ and any real $t$. In other words if $T_{t}$ is the "translation" $T_{t} \omega=\omega+t$ on $\Omega$ then $P=P \circ T_{t}^{-1}$ for any $t$.

The space $\Omega$ can be made into a Polish space (complete separable metric space) in which $\mathscr{F}$ is the $\sigma$-field generated by the open sets. This topology is the one induced by the vague topology for measures on $R$ if we identify each $\omega \in \Omega$ with the measure assigning mass 1 to each point of $\omega$. See [5] for details. As a consequence the existence of regular versions of conditional expectations will be taken for granted.

We shall assume throughout the present paper for all of our processes that the measure

$$
\mu(Q)=\int_{\Omega} N(\omega, Q) P(d \omega)
$$

is finite for bounded Borel $Q$. If the process is stationary, then $\alpha=\mu([0,1])$ is its intensity. 
As noted in [8], for fixed $A$ the Radon-Nikodym theorem implies the existence of a Borel function $P(A \mid x)(x \in R)$ such that

$$
\int_{A} N(\omega, Q) P(d \omega)=\int_{Q} P(A \mid x) \mu(d x) \quad(Q \in \mathscr{B})
$$

and $P(A \mid x)$ can be chosen so as to be a true probability in $A$ for fixed $x$. If in addition the process is stationary then $P(A \mid x)$ can be chosen so that $P(A \mid x)$ $=P(A+t \mid x+t)$ for all $A \in \mathscr{F}, x \in R, t \in R$; in this case we set $P_{0}(A)=P(A \mid 0)$ and call $P_{0}$ the Palm probability of the stationary point process. $P(A \mid x)$ can be interpreted as the conditional probability of $A$ given that the process has a point at $x$.

Consider now the space $R \times \Omega$ and the $\sigma$-field $\mathscr{B} \otimes \mathscr{F}$ in it. If we set

$$
m(Q \times A)=\int_{A} N(\omega, Q) P(d \omega)=\int_{Q} P(A \mid x) \mu(d x)
$$

then $m$ is a set function defined for rectangles $Q \times A(Q \in \mathscr{B}, A \in \mathscr{F})$, which can be extended to a measure $m$ on $\mathscr{B} \otimes \mathscr{F}$. By the Fubini theorem (see [3, p. 15]), if $D \in \mathscr{B} \otimes \mathscr{F}$ then

$$
m(D)=\int_{\Omega} N\left(\omega, D^{\omega}\right) P(d \omega)=\int_{R} P\left(D_{x} \mid x\right) \mu(d x)
$$

where $D_{x}=\{\omega \in \Omega:(x, \omega) \in D\}, D^{\omega}=\{x \in R:(x, \omega) \in D\}$. In [5] we show that when the process is stationary, practically all the formulae involving $P_{0}$ (such as (6) below) are immediate consequences of (4). We shall not go into this here. We note only that $P_{0}$ is carried by the set $\Omega_{0}=\{\omega \in \Omega: 0 \in \omega\}$. For proofs of many of the unproved statements of this section we refer the reader to [8], [5] and the literature cited in [5].

In the rest of this section we assume that $P$ is stationary. If $\omega \in \Omega$ and $x \in R$, we can enumerate the points of $\omega$

$$
\cdots<t_{-1}(\omega ; x)<t_{0}(\omega ; x)<t_{1}(\omega ; x)<\cdots
$$

so that $t_{0}(\omega ; x)<x \leqq t_{1}(\omega ; x)$. If $x=0$ we write simply $t_{i}(\omega)$ instead of $t_{i}(\omega ; 0)$. Then we set

$$
\zeta_{i}(\omega)=t_{i+1}(\omega)-t_{i}(\omega), \quad \theta(\omega)=-t_{0}(\omega)
$$

and for $\omega \in \Omega_{0}$,

$$
\eta_{i}(\omega)=\zeta_{i+1}(\omega) \quad\left(\omega \in \Omega_{0}\right) .
$$

The connection between $P$ and $P_{0}$ is known to be ([8])

$$
\begin{aligned}
\int_{\Omega} X(\omega) P(d \omega) & =\alpha \int_{\Omega_{0}} \int_{0}^{\eta_{0}(\omega)} X(\omega-x) d x P_{0}(d \omega) \\
& =\alpha \int_{0}^{\infty} \int_{\left\{\eta_{0} \geqq x\right\}} X(\omega-x) P_{0}(d \omega) d x .
\end{aligned}
$$


The probability $P_{0}$ is invariant on $\Omega_{0}$ under the one-to-one mapping $T^{*} \omega$ $=\omega-\eta_{0}(\omega)$ of $\Omega_{0}$ onto itself. In other words the $P_{0}$-distribution of the random sequence

$$
\ldots, \quad \eta_{-1}, \quad \eta_{0}, \quad \eta_{1}, \ldots
$$

is shift invariant. In particular if $i>0$ then

$$
\int_{\Omega} X(\omega) P(d \omega)=\alpha \int_{\Omega} \int_{\eta_{0}(\omega)+\cdots+\eta_{1-1}(\omega)}^{\eta_{0}(\omega)+\cdots+\eta_{i}(\omega)} X(\omega-x) d x P_{0}(d \omega)
$$

and analogously for $i<0$.

If $t \in R$ we let $\mathscr{F}_{t}$ denote the $\sigma$-field of events generated by the random variables $N(\omega, Q)$ with $Q \subseteq(-\infty, t)$. Note that $\mathscr{F}_{0}$ is clearly generated by $\theta(\omega), \zeta_{-1}(\omega)$, $\zeta_{-2}(\omega), \ldots$

We now introduce some notational conventions. Measures will sometimes be denoted by their corresponding "differentials", for instance

$$
P_{0}\left(\eta_{1} \in d x_{1}, \eta_{2} \in d x_{2}, \ldots\right)
$$

will denote the distribution of the sequence $\eta_{1}, \eta_{2}, \ldots$ in the space $R_{+}^{\infty}=R_{+} \times R_{+}$ $\times \cdots$ (where $\left.R_{+}=(0, \infty)\right)$, i.e. the probability measure $P_{0}\left(\left(\eta_{1}, \eta_{2}, \ldots\right) \in B\right)$ $\left(B \in \mathscr{B}^{\infty}\right)$. Analogously for all other measures. As is customary for random variables we shall frequently write $N(Q)$ for $N(\omega, Q), N[x, y)$ for $N(\omega,[x, y))$, etc. $E X$ and $E(X \mid \mathscr{D})$ will denote expectation and conditional expectation taken relative to $P$, while $E_{0} X$ and $E_{0}(X \mid \mathscr{D})$ will denote the corresponding expectations taken relative to $P_{0}$. When we want to exhibit $E(X \mid \mathscr{D})$ or $E_{0}(x \mid \mathscr{D})$ as random variables in $\omega$ we shall write $E(X \mid \mathscr{D})(\omega), E_{0}(X \mid \mathscr{D})(\omega)$. Finally $\lambda$ will denote the Lebesgue measure on $R$.

LEMMA 1. If $g\left(x, x_{1}, x_{2}, \ldots\right)$ is a bounded Borel function on $R_{+}^{\infty}$ then

$$
\begin{aligned}
\int_{\Omega} g\left(\theta(\omega), \zeta_{-1}(\omega), \zeta_{-2}(\omega), \ldots\right) P(d \omega) & \\
& =\alpha \int_{0}^{\infty} \int_{\Omega_{0}} \chi_{\left\{\eta_{0} \geqq x\right\}}(\omega) g\left(x, \eta_{-1}(\omega), \eta_{-2}(\omega), \ldots\right) P_{0}(d \omega) d x .
\end{aligned}
$$

This follows from (6) and the fact that if $\omega \in \Omega_{0}$ and $0<x \leqq \eta_{0}(\omega)$ then $\theta(\omega-x)=x$, $\zeta_{-1}(\omega-x)=\eta_{-1}(\omega), \zeta_{-2}(\omega-x)=\eta_{-2}(\omega)$, etc.

Lemma 2. If $G$ is a Borel set in $R_{+}^{\infty}$ then $P_{0}\left(\left(\eta_{-1}, \eta_{-2}, \ldots\right) \in G\right)=0$ if and only if the set $A=\left\{\omega \in \Omega:\left(t_{i}(\omega)-t_{i-1}(\omega), t_{i-1}(\omega)-t_{i-2}(\omega), \ldots\right) \in G\right.$ for some $\left.i\right\}$ is $a$ $P$-null event.

Proof. Let $A_{i}=\left\{\omega \in \Omega:\left(t_{i}(\omega)-t_{i-1}(\omega), t_{i-1}(\omega)-t_{i-2}(\omega), \ldots\right) \in G\right\}$. If

$$
P_{0}\left(\left(\eta_{-1}, \eta_{-2}, \ldots\right) \in G\right)=0
$$


then by (6) and Lemma 1

$$
\begin{aligned}
P\left(A_{0}\right) & =\int_{\Omega} \chi_{A_{0}}(\omega) P(d \omega) \\
& =\alpha \int_{0}^{\infty} \int_{\Omega_{0}} \chi_{\left\langle\eta_{0} \geqq x\right\}}(\omega) \chi_{A_{0}}(\omega-x) P_{0}(d \omega) d x \\
& =\alpha \int_{0}^{\infty} P_{0}\left(\eta_{0} \geqq x \text { and }\left(\eta_{-1}, \eta_{-2}, \ldots\right) \in G\right) d x=0 .
\end{aligned}
$$

For $A_{i}$ with $i>0$ the result follows from (8); analogously for $i<0$.

Conversely if $P(A)=0$ then $P\left(A_{0}\right)=0$, hence by the above equality

$$
P_{0}\left(\left(\eta_{0} \geqq x \text { and }\left(\eta_{-1}, \eta_{-2}, \ldots\right) \in G\right)=0 \text { for every } x>0\right. \text {. }
$$

It follows that $\left.P_{0}\left(\eta_{-1}, \eta_{-2}, \ldots\right) \in G\right)=0$.

3. The a.s. limit theorem. Let $[b, c)$ be a finite interval. If $\Delta=\left\{b=\xi_{0}<\xi_{1}<\cdots\right.$ $\left.<\xi_{n+1}=c\right\}$ is a partition of $[b, c)$, then $\|\Delta\|$ will denote the mesh of $\Delta$, i.e. the number $\max _{0 \leqq v \leqq n}\left(\xi_{v+1}-\xi_{v}\right)$. Suppose that to each partition $\Delta$ of $[b, c)$ corresponds a number $S_{\Delta}$. We shall say that $S_{\Delta}$ converges to $I$ as $\|\Delta\| \rightarrow 0$ (written $\lim _{\|\Delta\| \rightarrow 0} S_{\Delta}$ $=I$ ) if for every $\varepsilon>0$ there is a $\delta>0$ such that $\left\|S_{\Delta}-I\right\|<\varepsilon$ for all partitions $\Delta$ with $\|\Delta\|<\delta$. Note that $\lim _{\|\Delta\| \rightarrow 0} S_{\Delta}=I$ if and only if, for every sequence $\left\{\Delta_{k}\right\}$ with $\left\|\Delta_{k}\right\| \rightarrow 0, \lim _{k \rightarrow \infty} S_{\Delta_{k}}=I$.

The next definition is classical. An interval function $F[x, y)(x<y)$ is called Burkill integrable over the interval $[b, c)$ if

$$
\lim _{\|\Delta\| \rightarrow 0} \sum_{v=0}^{n} F\left[\xi_{v}, \xi_{v+1}\right)=W_{b}^{c}
$$

exists. $W_{b}^{c}$ is then called the Burkill integral of $F$ over $[b, c)$.

The aim of the present section is to prove the following theorem.

THEOREM 1. There is a "canonical" version $Z(\omega,[x, y))(\omega \in \Omega, x<y)$ of the random interval function $E\left(N[x, y) \mid \mathscr{F}_{x}\right)(\omega)$, such that for every $\omega \in \Omega$ the sample interval function $Z(\omega,[x, y))(x<y)$ is Burkill integrable over any finite interval.

We shall denote the Burkill integral of $Z(\omega,[\cdot, \cdot))$ over $[b, c)$ by $W(\omega,[b, c))$. The latter is identified in the proof. For fixed $\omega, W(\omega,[b, c))$ is a $\sigma$-additive function on the class of left-closed right-open intervals (see $\$ 5$ ).

We first choose a regular version of $P_{0}\left(A \mid \eta_{-1}=x_{1}, \eta_{-2}=x_{2}, \ldots\right)$, i.e. one which is a true probability in $A$ for fixed $\left(x_{1}, x_{2}, \ldots\right) \in R_{+}^{\infty}$ and Borel measurable in $\left(x_{1}, x_{2}, \ldots\right) \in R_{+}^{\infty}$ for each fixed $A \in \mathscr{F}$. This version will be fixed throughout this paper. Next we establish the relationship between $P$ and $P_{0}$ on $\mathscr{F}_{0}$. 
DEFINITION.

$$
\psi\left(x, x_{1}, x_{2}, \ldots\right)=P_{0}\left(\eta_{0} \geqq x \mid \eta_{-1}=x_{1}, \eta_{-2}=x_{2}, \ldots\right) .
$$

This is a Borel function on $R_{+}^{\infty}$, being left continuous in $x$ for fixed $\left(x_{1}, x_{2}, \ldots\right)$.

\section{LEMMA 3.}

$$
\begin{aligned}
P\left(\theta \in d x, \zeta_{-1} \in d x_{1}, \zeta_{-2} \in d x_{2}, \ldots\right) & \\
& =\alpha \psi\left(x, x_{1}, x_{2}, \ldots\right) \cdot d x \cdot P_{0}\left(\eta_{-1} \in d x_{1}, \eta_{-2} \in d x_{2}, \ldots\right)
\end{aligned}
$$

Proof. If $g\left(x, x_{1}, x_{2}, \ldots\right)$ is a bounded Borel function on $R_{+}^{\infty}$ then using the transformation $\Omega \ni \omega \Rightarrow\left(\theta(\omega), \zeta_{-1}(\omega), \ldots\right) \in R_{+}^{\infty}$ we have by $(6)$ and Lemma 1

$$
\begin{aligned}
\int_{R_{+}^{\infty}} g\left(x, x_{1}, x_{2}, \ldots\right) P\left(\theta \in d x, \zeta_{-1} \in d x_{1}, \ldots\right) \\
\quad=\int_{\Omega} g\left(\theta(\omega), \zeta_{-1}(\omega), \ldots\right) P(d \omega) \\
=\alpha \int_{0}^{\infty} \int_{\Omega_{0}} \chi_{\left(\eta_{0} \geqq x\right)}(\omega) \cdot g\left(x, \eta_{-1}(\omega), \ldots\right) P_{0}(d \omega) d x \\
=\alpha \int_{0}^{\infty} \int_{\Omega_{0}} g\left(x, \eta_{-1}(\omega), \ldots\right) \cdot E\left(\chi_{\left(\eta_{0} \geqq x\right)} \mid \eta_{-1}, \eta_{-2}, \ldots\right) P_{0}(d \omega) d x \\
=\alpha \int_{0}^{\infty} \int_{R_{+}^{\infty}} g\left(x, x_{1}, x_{2}, \ldots\right) P\left(\eta_{0} \geqq x \mid \eta_{-1}=x_{1}, \eta_{-2}=x_{2}, \ldots\right) \\
P_{0}\left(\eta_{-1} \in d x_{1}, \eta_{-2} \in d x_{2}, \ldots\right) d x
\end{aligned}
$$

from which the result follows.

Lemma 4. If $A \in \mathscr{F}$ then for $d x \cdot P_{0}\left(\eta_{-1} \in d x_{1}, \ldots\right)$-almost all $\left(x, x_{1}, x_{2}, \ldots\right)$ $\in R_{+}^{\infty}$, we have

$$
\begin{aligned}
P\left(A \mid \theta=x, \zeta_{-1}=\right. & \left.x_{1}, \zeta_{-2}=x_{2}, \ldots\right) \psi\left(x, x_{1}, x_{2}, \ldots\right) \\
& =P_{0}\left((A+x) \cap\left\{\eta_{0} \geqq x\right\} \mid \eta_{-1}=x_{1}, \eta_{-2}=x_{2}, \ldots\right) .
\end{aligned}
$$

(If we write $\pi_{x_{1} x_{2} \ldots}(\cdot)$ for the probability $P_{0}\left(\cdot \mid \eta_{-1}=x_{1}, \eta_{-2}=x_{2}, \ldots\right)$, this lemma simply says that $P\left(A \mid \theta=x, \zeta_{-1}=x_{1}, \ldots\right)=\pi_{x_{1} x_{2} \ldots}\left(A+x \mid \eta_{0} \geqq x\right)$.)

Proof. Let $Q$ be a Borel set in $(0, \infty)$ and $S$ a Borel set in $R_{+}^{\infty}$. We start out with the integral

$$
\int_{Q \times S} P\left(A \mid \theta=x, \zeta_{-1}=x_{1}, \ldots\right) P\left(\theta \in d x, \zeta_{-1} \in d x_{1}, \ldots\right) .
$$

By Lemma 3 this is equal to

(10) $\int_{Q \times S} P\left(A \mid \theta=x, \zeta_{-1}=x_{1}, \ldots\right) \cdot \alpha \psi\left(x, x_{1}, \ldots\right) d x P_{0}\left(\eta_{-1} \in d x_{1}, \ldots\right)$. 
On the other hand (9) is equal to

$$
\begin{aligned}
\int_{R_{+}^{\infty}} \chi_{Q}(x) \cdot \chi_{S}\left(x_{1}, x_{2}, \ldots\right) P\left(A \mid \theta=x, \zeta_{-1}=x_{1}, \ldots\right) P\left(\theta \in d x, \zeta_{-1} \in d x_{1}, \ldots\right) \\
\quad=\int_{\Omega} \chi_{Q}(\theta(\omega)) \cdot \chi_{S}\left(\zeta_{-1}(\omega), \zeta_{-2}(\omega), \ldots\right) P\left(A \mid \theta, \zeta_{-1}, \ldots\right)(\omega) P(d \omega) \\
\quad=\int_{\Omega} \chi_{Q}(\theta(\omega)) \cdot \chi_{S}\left(\zeta_{-1}(\omega), \ldots\right) \chi_{A}(\omega) P(d \omega) \\
\quad=\alpha \int_{0}^{\infty} \int_{\Omega_{0}} \chi_{\left\{\eta_{0} \geqq x\right\}}(\omega) \cdot \chi_{Q}(x) \chi_{S}\left(\eta_{-1}(\omega), \ldots\right) \chi_{A}(\omega-x) P_{0}(d \omega) d x \quad(\text { by }(6)) \\
\quad=\alpha \int_{0}^{\infty} \chi_{Q}(x) \int_{\Omega_{0}} \chi_{S}\left(\eta_{-1}(\omega), \ldots\right) \chi_{A+x}(\omega) \chi_{\left(\eta_{0} \geqq x\right\}}(\omega) P_{0}(d \omega) d x \\
\quad=\alpha \int_{0}^{\infty} \chi_{Q}(x) \int_{\Omega_{0}} \chi_{S}\left(\eta \eta_{-1}(\omega), \ldots\right) \cdot P_{0}\left((A+x) \cap\left\{\eta_{0} \geqq x\right\} \mid \eta_{-1}, \ldots\right)(\omega) \\
=\alpha \int_{0}^{\infty} \chi_{Q}(x) \int_{R_{+}^{\infty}} \chi_{S}\left(x_{1}, x_{2}, \ldots\right) P_{0}\left((A+x) \cap\left\{\eta_{0} \geqq x\right\} \mid \eta_{-1}=x_{1}, \ldots\right) \\
\quad=\alpha \int_{Q} \int_{S} P_{0}\left((A+x) \cap\left\{\eta_{0} \geqq x\right\} \mid \eta_{-1}=x_{1}, \ldots\right) P_{0}\left(\eta_{-1} \in d x_{1}, \ldots\right) d x .
\end{aligned}
$$

The result follows if we compare this with (10).

LEMma 5. The event $H_{1}=\left\{\omega \in \Omega: \psi\left(t_{i}(\omega)-t_{i-1}(\omega), t_{i-1}(\omega)-t_{i-2}(\omega), \ldots\right)>0\right.$ for all $i$ \} has $P$-measure 1.

Proof. Let $G=\left\{\left(x, x_{1}, \ldots\right): \psi\left(x, x_{1}, \ldots\right)=0\right\}$ and for each $\left(x_{1}, x_{2}, \ldots\right)$ define $G_{x_{1} x_{2} \ldots}=\left\{x: \psi\left(x, x_{1}, x_{2}, \ldots\right)=0\right\}$. From the definition of $\psi$ we see that if for each $\left(x_{1}, x_{2}, \ldots\right)$ we let

$$
\gamma\left(x_{1}, x_{2}, \ldots\right)=\sup \left\{x: P_{0}\left(\eta_{0} \geqq x \mid \eta_{-1}=x_{1}, \ldots\right)>0\right\}
$$

then $G_{x_{1} x_{2} \ldots}$ is $\left[\gamma\left(x_{1}, x_{2}, \ldots\right), \infty\right)$ or $\left(\gamma\left(x_{1}, x_{2}, \ldots\right), \infty\right)$. In either case we clearly have for all $x_{1}, x_{2}, \ldots$

$$
P_{0}\left(\eta_{0} \in G_{x_{1} x_{2} \ldots} \mid \eta_{-1}=x_{1}, \ldots\right)=0
$$

hence $P_{0}\left(\left(\eta_{0}, \eta_{-1}, \ldots\right) \in G\right)=0, P_{0}\left(\left(\eta_{-1}, \eta_{-2}, \ldots\right) \in G\right)=0$ and the assertion follows from Lemma 2.

LEMMA 6. If $X(\omega)$ is an integrable random variable and if we set $X_{t}(\omega)=X(\omega+t)$ $(t \in R)$, then $E\left(X_{t} \mid \mathscr{F}_{0}\right)(\omega-t)$ is a version of $E\left(X \mid \mathscr{F}_{t}\right)(\omega)$. 
This follows easily from the stationariness of $P$. We leave the proof to the reader. Combining this with Lemma 4 we get canonical versions of our conditional expectations.

Lemma 7. Given $x \in R$ and an integrable random variable $X(\omega)$ there is a $P$-null set $E$ (depending on $x$ and $X(\omega)$ ) in $\Omega$ with the following property. If $\omega_{0} \notin E$ and if

$$
\begin{array}{r}
t_{0}\left(\omega_{0} ; x\right)=b_{0} \\
t_{0}\left(\omega_{0} ; x\right)-t_{-1}\left(\omega_{0} ; x\right)=x_{1} \\
t_{-1}\left(\omega_{0} ; x\right)-t_{-2}\left(\omega_{0} ; x\right)=x_{2}
\end{array}
$$

then

$$
\begin{aligned}
& E\left(X \mid \mathscr{F}_{x}\right)\left(\omega_{0}\right) \\
& \quad=\frac{1}{\psi\left(x-b_{0}, x_{1}, x_{2}, \ldots\right)} \int_{\left\{\eta_{0} \geqq x-b_{0}\right\}} X\left(\omega+b_{0}\right) P_{0}\left(d \omega \mid \eta_{-1}=x_{1}, \ldots\right) .
\end{aligned}
$$

Lemma 8. For $P_{0}\left(\eta_{-1} \in d x_{1}, \eta_{-2} \in d x_{2}, \ldots\right)$-almost all $\left(x_{1}, x_{2}, \ldots\right) \in R_{+}^{\infty}$,

$$
\int_{\Omega_{0}} N(\omega,(b, c)) P_{0}\left(d \omega \mid \eta_{-1}=x_{1}, \eta_{-2}=x_{2}, \ldots\right)<\infty
$$

for any finite interval $(b, c)$.

Note. This lemma is needed because $\int_{\Omega_{0}} N(\omega,(b, c)) P_{0}(d \omega)$ may be infinite for all $(b, c)$. Let for instance $\eta_{0}$ be a random variable with $P_{0}\left(\eta_{0}=1 / n\right)=$ const $/ n^{2}$, set $\eta_{i}=\eta_{0}(i=0, \pm 1, \ldots)$ and construct the corresponding stationary point process.

Proof. We first show that for every $x>0$,

$$
\left.\int_{\{\eta-1 \geqq x\}} N(\omega,(b, c)) P_{0}(d \omega)<\infty \quad \text { (any } b<c\right) .
$$

In fact $\int_{\Omega} N(\omega,(b, c+1)) P(d \omega)<\infty$ and by a relation analogous to (6) (see (8)), this integral is equal to

$$
\begin{aligned}
\alpha \int_{\Omega_{0}} \int_{0}^{\eta_{-1}(\omega)} N(\omega+x,(b, c+1)) d x P_{0}(d \omega) & \\
& =\alpha \int_{0}^{\infty} \int_{\left\{\eta_{-1} \geqq x\right\}} N(\omega,(b-x, c+1-x)) P_{0}(d \omega) d x .
\end{aligned}
$$

Hence for almost all $x>0, \int_{\left\{\eta_{-1} \geqq x\right\}} N(\omega,(b-x, c+1-x)) P_{0}(d \omega)<\infty$. When $x \in(0,1)$ the interval $(b, c)$ is contained in $(b-x, c+1-x)$, therefore

$$
\int_{\left\{\eta_{-1} \geq x\right\}} N(\omega,(b, c)) P_{0}(d \omega)<\infty
$$

for almost all $x \in(0,1)$ and, therefore, (by monotonicity) for all $x>0$. 
If now $X(\omega)$ is any $P_{0}$-integrable random variable then

$$
\begin{aligned}
\int_{[x, \infty) \times R_{+} \times R_{+} \times \ldots} & \int_{\Omega_{0}} X(\omega) P_{0}\left(d \omega \mid \eta_{-1}=x_{1}, \ldots\right) P_{0}\left(\eta_{-1} \in d x_{1}, \ldots\right) \\
& =\int_{[x, \infty) \times R_{+} \times \ldots} E_{0}\left(X \mid \eta_{-1}=x_{1}, \ldots\right) P_{0}\left(\eta_{-1} \in d x_{1}, \ldots\right) \\
& =\int_{\left\{\eta_{-1} \geqq x\right\}} E_{0}\left(X \mid \eta_{-1}, \ldots\right)(\omega) P_{0}(d \omega)=\int_{\left\{\eta_{-1} \geqq x\right\}} X(\omega) P_{0}(d \omega) .
\end{aligned}
$$

Taking a sequence $X_{n}(\omega) \uparrow N(\omega,(b, c))$ we see that

$$
\begin{aligned}
& \int_{[x, \infty) \times R_{+} \times \ldots} \int_{\Omega_{0}} N(\omega,(b, c)) P_{0}\left(d \omega \mid \eta_{-1}=x_{1}, \ldots\right) P_{0}\left(\eta_{-1} \in d x_{1}, \ldots\right) \\
&=\int_{\left\{\eta_{-1} \geqq x\right\}} N(\omega,(b, c)) P_{0}(d \omega)<\infty \quad \text { by }(12),
\end{aligned}
$$

i.e. $\int_{\Omega_{j}} N(\omega,(b, c)) P_{0}\left(d \omega \mid \eta_{-1}=x_{1}, \ldots\right)<\infty$ for $P_{0}\left(\eta_{-1} \in d x_{1}, \ldots\right)$-almost all $\left(x_{1}, x_{2}, \ldots\right)$. The result follows if we let $b, c$ run through all pairs of integers $k, l$ with $k<l$.

If now $G^{*}=\left\{\left(x_{1}, x_{2}, \ldots\right): \int_{\Omega_{0}} N(\omega,(b, c)) P_{0}\left(d \omega \mid \eta_{-1}=x_{1}, \ldots\right)=\infty\right.$ for some finite interval $(b, c)\}$ then $G^{*}$ is a null set under $P_{0}\left(\eta_{-1} \in d x_{1}, \ldots\right)$ and hence by Lemma 2 the event

$$
H_{2}=\left\{\omega \in \Omega:\left(t_{i}(\omega)-t_{i-1}(\omega), t_{i-1}(\omega)-t_{i-2}(\omega), \ldots\right) \notin G^{*}, \text { any } i\right\}
$$

has $P$-measure 1. Let $H_{1}$ be as in Lemma 5 .

Definition. If $x \in R$ and $0 \leqq X(\omega) \leqq N(\omega,[b, c))$ for some $b, c$, we define the canonical version of $E\left(X \mid \mathscr{F}_{x}\right)$ as follows: If $\omega_{0} \notin H_{1} \cap H_{2}$ then $E\left(X \mid \mathscr{F}_{x}\right)\left(\omega_{0}\right)=0$; if $\omega_{0} \in H_{1} \cap H_{2}$ then $E\left(X \mid \mathscr{F}_{x}\right)\left(\omega_{0}\right)$ is as in (11). In particular the canonical versions of $E\left(N[x, y) \mid \mathscr{F}_{x}\right)\left(\omega_{0}\right)$ and $P\left(N[x, y) \geqq 1 \mid \mathscr{F}_{x}\right)\left(\omega_{0}\right)$ are given, for $\omega_{0} \in H_{1} \cap H_{2}$, by

$$
\frac{1}{\psi\left(x-b_{0}, x_{1}, x_{2}, \ldots\right)} \int_{\left\{x-b_{0} \leqq \eta_{0}<y-b_{0}\right\}} N\left(\omega,\left[x-b_{0}, y-b_{0}\right)\right) P_{0}\left(d \omega \mid \eta_{-1}=x_{1}, \ldots\right)
$$

and

$$
\left(\psi\left(x-b_{0}, x_{1}, x_{2}, \ldots\right)\right)^{-1} P_{0}\left(x-b_{0} \leqq \eta_{0}<y-b_{0} \mid \eta_{-1}=x_{1}, \ldots\right)
$$

respectively (we use the notation of Lemma 7). We shall write $Z\left(\omega_{0},[x, y)\right)$ for the canonical version of $E\left(N[x, y) \mid \mathscr{F}_{x}\right)\left(\omega_{0}\right)$.

Proof of Theorem 1. We shall prove that if $\omega_{0} \in H_{1} \cap H_{2}$ then the numerical interval function $Z\left(\omega_{0},[x, y)\right)(x<y)$ is Burkill integrable over any finite interval.

Fix $\omega_{0} \in H_{1} \cap H_{2}$. If $\omega_{0}$ is the set $\cdots<t_{-1}\left(\omega_{0}\right)<t_{0}\left(\omega_{0}\right)<t_{1}\left(\omega_{0}\right)<\cdots$ where $t_{0}\left(\omega_{0}\right)<0 \leqq t_{1}\left(\omega_{0}\right)$, we shall show that $Z\left(\omega_{0},[x, y)\right)$ is Burkill integrable over any interval $\left[t_{i}\left(\omega_{0}\right), t_{i+1}\left(\omega_{0}\right)\right)$. Fix such an interval and let $b_{0}=t_{i}\left(\omega_{0}\right)<c_{0} \leqq t_{i+1}\left(\omega_{0}\right)$. If $b_{0}<x<c_{0}$ then clearly $t_{0}\left(\omega_{0} ; x\right)=b_{0}$ and we set

$$
t_{0}\left(\omega_{0} ; x\right)-t_{-1}\left(\omega_{0} ; x\right)=x_{1}, \quad t_{-1}\left(\omega_{0} ; x\right)-t_{-2}\left(\omega_{0} ; x\right)=x_{2}, \ldots
$$


Now consider a partition $\Delta=\left\{b_{0}=\xi_{0}<\xi_{1}<\cdots<\xi_{n}<\xi_{n+1}=c_{0}\right\}$ of $\left[b_{0}, c_{0}\right)$. By definition

$$
\begin{aligned}
& \sum_{v=0}^{n} Z\left(\omega_{0},\left[\xi_{v}, \xi_{v+1}\right)\right)=Z\left(\omega_{0},\left[b_{0}, \xi_{1}\right)\right)+\sum_{v=1}^{n} Z\left(\omega_{0},\left[\xi_{v}, \xi_{v+1}\right)\right) \\
&=\frac{1}{\psi\left(x_{1}, x_{2}, \ldots\right)} \int_{\left\{x_{1} \leqq \eta_{0}<x_{1}+\xi_{1}-b_{0}\right\}} \begin{array}{c}
N\left(\omega,\left[x_{1}, x_{1}+\xi_{1}-b_{0}\right)\right) \\
P_{0}\left(d \omega \mid \eta_{-1}=x_{2}, \eta_{-2}=x_{3}, \ldots\right)
\end{array} \\
&+\sum_{v=1}^{n} \frac{1}{\psi\left(\xi_{v}-b_{0}, x_{1}, x_{2}, \ldots\right)} \int_{\left\{\xi_{v}-b_{0} \leqq \eta_{0}<\xi_{v+1}-b_{0}\right\}} N\left(\omega,\left[\xi_{v}-b_{0}, \xi_{v+1}-b_{0}\right)\right) \\
& P_{0}\left(d \omega \mid \eta_{-1}=x_{1}, \eta_{-2}=x_{2}, \ldots\right) .
\end{aligned}
$$

When $\|\Delta\| \rightarrow 0, \xi_{1} \rightarrow b_{0}$ and hence the first term converges to

$$
\begin{aligned}
\frac{1}{\psi\left(x_{1}, x_{2}, \ldots\right)} \int_{\left\{\eta_{0}=x_{1}\right\}} N\left(\omega,\left\{x_{1}\right\}\right) P_{0}\left(d \omega \mid \eta_{-1}=x_{2}, \eta_{-2}=x_{3}, \ldots\right) \\
\quad=\left(\psi\left(x_{1}, x_{2}, \ldots\right)\right)^{-1} P_{0}\left(\eta_{0}=x_{1} \mid \eta_{-1}=x_{2}, \eta_{-2}=x_{3}, \ldots\right)
\end{aligned}
$$

Since $b_{0}, x_{1}, x_{2}, \ldots$ are fixed we simplify the notation for the remaining terms by setting

$$
\begin{aligned}
\pi(\cdot) & =P_{0}\left(\cdot \mid \eta_{-1}=x_{1}, \eta_{-2}=x_{2}, \ldots\right), \\
F(x) & =\pi\left(\eta_{0}<x\right), \\
\psi\left(x-b_{0}\right) & =\psi\left(x-b_{0}, x_{1}, x_{2}, \ldots\right) .
\end{aligned}
$$

Note that $1-F\left(x-b_{0}\right)=\pi\left(\eta_{0} \geqq x-b_{0}\right)=\psi\left(x-b_{0}\right)$. Since $\omega_{0} \in H_{1}$ we have $\psi\left(c-b_{0}\right)$ $>0$ and if we let $\beta=\bar{\psi}\left(c-b_{0}\right)$ then $\psi\left(x-b_{0}\right) \geqq \beta$ for all $x \in\left[b_{0}, c_{0}\right)$. Also $\omega \in H_{2}$ implies

$$
\int_{\Omega_{0}} N\left(\omega,\left[b_{0}, c_{0}\right)\right) \pi(d \omega)<\infty
$$

Then

$$
\begin{aligned}
& \sum_{v=1}^{n} \frac{1}{\overline{\psi\left(\xi_{v}-b_{0}\right)}} \int_{\left\{\xi_{v}-b_{0} \leqq \eta_{0}<\xi_{v+1}-b_{0}\right\}} N\left(\omega,\left[\xi_{v}-b_{0}, \xi_{v+1}-b_{0}\right)\right) \pi(d \omega) \\
& =\sum_{v=1}^{n} \frac{1}{\bar{\psi}\left(\xi_{v}-b_{0}\right)} \pi\left(\xi_{v}-b_{0} \leqq \eta_{0}<\xi_{v+1}-b_{0}\right) \\
& +\sum_{\nu=1}^{n} \frac{1}{\bar{\psi}\left(\xi_{v}-b_{0}\right)} \int_{\left\{\xi_{v}-b_{0} \leqq \eta_{0}<\xi_{v+1}-b_{0}\right\}}\left[N\left(\omega,\left[\xi_{v}-b_{0}, \xi_{v+1}-b_{0}\right)\right)-1\right] \pi(d \omega) .
\end{aligned}
$$

The first sum on the right is equal to

$$
\sum_{v=1}^{n} \frac{1}{1-F\left(\xi_{v}-b_{0}\right)} \cdot\left(F\left(\xi_{v+1}-b_{0}\right)-F\left(\xi_{v}-b_{0}\right)\right)=\int_{\left(0, c_{0}-b_{0}\right)} f_{n}(x) d F(x)
$$

where $f_{n}(x)$ is the step function assuming the value 0 in $\left(0, \xi_{1}-b_{0}\right)$ and the value $1 /\left(1-F\left(\xi_{v}-b_{0}\right)\right)$ in $\left[\xi_{v}-b_{0}, \xi_{v+1}-b_{0}\right)(\nu=1,2, \ldots, n)$. The integral is meant in the 
Lebesgue-Stieltjes sense, i.e. $d F(x)$ is the measure induced by $F$ on $(0, \infty)$. Clearly, when $\|\Delta\| \rightarrow 0, \lim _{n \rightarrow \infty} f_{n}(x)=1 /(1-F(x))$ by the left continuity of $1 /(1-F(x))$, hence by the Lebesgue dominated convergence theorem the first sum converges to

$$
\int_{\left(0, c_{0}-b_{0}\right)} \frac{F(d x)}{1-F(x)}
$$

If $F$ has no jumps this is $-\log \left(1-F\left(c_{0}-b_{0}\right)\right)$.

The second sum on the right of (17) (which is nonnegative since the integrand is $\geqq 0$ on the region of integration) is less than or equal to

$$
\frac{1}{\beta} \int_{\Omega_{0}}\left(\sum_{\nu=1}^{n} N\left(\omega,\left[\xi_{v}-b_{0}, \xi_{v+1}-b_{0}\right)\right) \cdot \chi_{\left\{N\left(\xi_{v}-b_{0}, \xi_{v+1}-b_{0}\right) \geqq 2\right\}}(\omega) \pi(d \omega) .\right.
$$

When $\|\Delta\| \rightarrow 0$ the integrand in (20) converges to zero pointwise (in fact for each $\omega$ the integrand is zero when $\|\Delta\|$ is less than the minimal distance between the points of $\omega$ in $\left.\left(b_{0}, c_{0}\right)\right)$. Since the integrand is dominated by $N\left(\omega,\left(b_{0}, c_{0}\right)\right)$, the Lebesgue dominated convergence theorem (see [1]) together with (16) implies that (20) converges to zero. (Note that the filter we obtain when we direct partitions according to their mesh has a denumerable base.) This completes the proof.

Note that when $b_{0}=t_{i}\left(\omega_{0}\right)<c_{0} \leqq t_{i+1}\left(\omega_{0}\right)$ we have identified the limit $W\left(\omega_{0},\left[b_{0}, c_{0}\right)\right)$ as the sum of (15) and (19). For the general interval $[b, c)$ a finite sum of such expressions is needed.

Corollary. Suppose $\Delta_{k}=\left\{b=\xi_{0}^{k}<\xi_{1}^{k}<\cdots<\xi_{n_{k}+1}^{k}=c\right\}, k=1,2, \ldots$, is a sequence of partitions of $[b, c)$ such that $\left\|\Delta_{k}\right\| \rightarrow 0$. Using any versions of the conditional expectations define

$$
S_{k}(\omega)=\sum_{v=0}^{n_{k}} E\left(N\left[\xi_{v}^{k}, \xi_{v+1}^{k}\right) \mid \mathscr{F}_{\xi_{v}^{k}}\right)
$$

Then

$$
\lim _{k \rightarrow \infty} S_{k}(\omega)=W(\omega,[b, c)) \text { a.s. }
$$

We now turn to the local limit theorem.

LEMma 9. For every $\omega \in \Omega, Z(\omega,[x, y))$ is continuous from the left in the halfplane $\{(x, y): x<y\}$ in the sense that if $u \uparrow x$ and $v \uparrow y$ then $Z(\omega,[u, v)) \rightarrow Z(\omega,[x, y))$. In particular $Z(\omega,[x, y))$ is jointly measurable in $(\omega, x, y)$ relative to the $\sigma$-field $\mathscr{F} \otimes \mathscr{B} \otimes \mathscr{B}$.

Proof. For each $\omega, N(\omega,[x, y))$ is continuous from the left, i.e. if $u \uparrow x$ and $v \uparrow y$ then $N(\omega,[u, v)) \rightarrow N(\omega,[x, y))$. If now $\omega_{0} \in H_{1} \cap H_{2}, t_{0}\left(\omega_{0} ; x\right)=b_{0}$ and $u \uparrow x$ then $t_{0}\left(\omega_{0} ; u\right)=b_{0}$ eventually, hence by (13), Lemma 8 and the Lebesgue dominated convergence theorem, $Z\left(\omega_{0},[x, y)\right)$ is left continuous. 
THEOREM 2. The limit

$$
Y(\omega)=\lim _{[x, y] \downarrow\{0\}} \frac{Z(\omega,[x, y))}{y-x}
$$

exists almost surely and $E Y \leqq \alpha .([x, y] \downarrow\{0\}$ means $x \leqq 0, y \geqq 0, y-x>0, x \rightarrow 0$, $y \rightarrow 0$.)

Proof. For fixed $\omega$ the function $f(t)=W(\omega,[0, t))$ is nondecreasing, hence $f^{\prime}(t)=\lim _{[u, v] \downarrow\{t\}} W(\omega,[u, v)) /(v-u)$ exists for almost all $t>0$ (recall that $W$ is additive). By a well-known theorem on Burkill integrals (see for instance [7, p. 23]) this is equal, for almost all $t>0$, to the corresponding limit for $Z$. The Fubini theorem together with Lemma 9 implies the existence of a $t>0$ such that the limit exists almost surely at that $t$. The theorem follows from the stationariness of the process.

4. The mean theorem. We begin with a lemma.

LEMMA 10. Suppose $\omega_{0} \in \Omega$ and $b<c$. If $\omega_{0}$ has no points in $[b, c)$ and if $\Delta_{k}=\left\{b=\xi_{0}^{k}<\xi_{1}^{k}<\cdots<\xi_{n_{k}+1}^{k}=c\right\}, k=1,2, \ldots$, is a sequence of partitions of $[b, c)$ such that $\Delta_{k+1}$ is a refinement of $\Delta_{k}$, then the sequence

$$
S_{k}\left(\omega_{0}\right)=\sum_{\nu=0}^{n_{k}} P\left(N\left[\xi_{v}, \xi_{v+1}\right) \geqq 1 \mid \mathscr{F}_{\xi_{v}}\right)\left(\omega_{0}\right)
$$

(where we use canonical versions on the right) is nondecreasing.

Proof. This follows from (14).

THEOREM 3. If for each partition $\Delta=\left\{b=\xi_{0}<\xi_{1}<\cdots<\xi_{n+1}=c\right\}$ of a given interval $[b, c)$ we let $S_{\Delta}(\omega)=\sum_{v=0}^{n} E\left(N\left[\xi_{v}, \xi_{v+1}\right) \mid \mathscr{F}_{\xi_{v}}\right)$ then

$$
\lim _{\|\Delta\| \rightarrow 0} \int_{\Omega}\left|S_{\Delta}(\omega)-W(\omega,[b, c))\right| P(d \omega)=0
$$

Proof. For the sake of simplicity we assume $b=0, c=1$ and write $W(\omega)$ for $W(\omega,[0,1))$. Then $\int_{\Omega} S_{\Delta}(\omega) P(d \omega)=E(N[0,1))=\alpha$ and therefore by the corollary to Theorem 1 we have $\int_{\Omega} W(\omega) P(d \omega) \leqq \alpha$. Since $S_{\Delta} \geqq 0$, to show (21) it is sufficient, by a well-known theorem, to prove

$$
\int_{\Omega} W(\omega) P(d \omega)=\alpha .
$$

To do this we take a sequence $\Delta_{k}=\left\{0=\xi_{0}^{k}<\xi_{1}^{k}<\cdots<\xi_{n_{k}+1}^{k}=1\right\}, k=1,2, \ldots$, of partitions such that $\Delta_{k+1}$ is a refinement of $\Delta_{k}$ and $\left\|\Delta_{k}\right\| \rightarrow 0$, and prove that the sequence $S_{\Delta_{k}}$ is uniformly integrable. This will imply mean convergence of $S_{\Delta_{k}}$ to 
$W$ and hence also (22). Using canonical versions we have

$$
\begin{aligned}
S_{\Delta_{k}}(\omega)= & \sum_{v=0}^{n_{k}} E\left(N\left[\xi_{v}^{k}, \xi_{v+1}^{k}\right) \mid \mathscr{F}_{\xi_{v}^{k}}\right)(\omega) \\
= & \sum_{\nu=0}^{n_{k}} E\left(N\left[\xi_{v}^{k}, \xi_{v+1}^{k}\right) \cdot \chi_{\left(N\left[\xi_{v}^{k}, \xi_{v+1}^{k}\right) \geqq 1\right\}} \mid \mathscr{F}_{\xi_{v}}^{k}\right)(\omega) \\
= & \left.\sum_{\nu=0}^{n_{k}} E\left(N\left[\xi_{v}^{k}, \xi_{v+1}^{k}\right)-1\right) \cdot \chi_{\left(N\left\{\xi_{v}^{k}, \xi_{v+1}^{k}\right) \geqq 1\right\}} \mid \mathscr{F}_{\xi_{v}^{k}}^{k}\right)(\omega) \\
& +\sum_{v=0}^{n_{k}} E\left(\chi_{\left(N\left[\xi_{v}^{k}, \xi_{v+1}^{k}\right) \geqq 1\right\}} \mid \mathscr{F}_{\xi_{v}^{k}}\right)(\omega)=A_{k}(\omega)+B_{k}(\omega) \text { say. }
\end{aligned}
$$

Now

$$
\left(N\left(\xi_{v}^{k}, \xi_{v+1}^{k}\right)-1\right) \cdot \chi_{\left(N\left[\xi_{v}^{k}, \xi_{v+1}^{k}\right) \geqq 1\right\}} \leqq N\left[\xi_{v}^{k}, \xi_{v+1}^{k}\right) \cdot \chi_{\left\{N\left\{\xi_{v}^{k}, \xi_{v+1}^{k} \geqq 2\right\}\right.}
$$

hence

$$
\int_{\Omega} A_{k}(\omega) P(d \omega) \leqq \int_{\Omega} \sum_{\nu=0}^{n_{k}} N\left[\xi_{v}^{k}, \xi_{v+1}^{k}\right) \chi_{\left(N\left[\xi_{v}^{k}, \xi_{v+1}^{k}\right) \geqq 2\right\}} P(d \omega)
$$

which converges to zero because the integrand converges pointwise to zero and is dominated by $N[0,1)$.

On the other hand

$$
\begin{aligned}
B_{k}(\omega)= & \sum_{\nu=0}^{n_{k}} P\left(N\left[\xi_{v}^{k}, \xi_{v+1}^{k}\right) \geqq 1 \mid \mathscr{F}_{\xi_{v}^{k}}\right)(\omega) \\
= & \sum_{v=0}^{n_{k}} P\left(N\left[\xi_{v}^{k}, \xi_{v+1}^{k}\right) \geqq 1 \mid \mathscr{F}_{\xi_{v}^{k}}\right)(\omega) \cdot \chi_{\left\{N\left[\xi_{v}^{k}, \xi_{v+1}^{k}\right) \geqq 1\right\}}(\omega) \\
& +\sum_{\nu=0}^{n_{k}} P\left(N\left[\xi_{v}^{k}, \xi_{v+1}^{k}\right) \geqq 1 \mid \mathscr{F}_{\xi_{v}^{k}}\right)(\omega) \cdot \chi_{\left\{N\left\{\xi_{v}^{k}, \xi_{v+1}^{k}\right)=0\right\}}(\omega) \\
= & C_{k}(\omega)+E_{k}(\omega) \text { say. }
\end{aligned}
$$

Since $C_{k}(\omega) \leqq \sum_{v=0}^{n_{k}} \chi_{\left\{N\left[\xi_{v}^{k}, \xi_{v+1}^{k}\right) \geqq 1\right\}}(\omega) \leqq N(\omega,[0,1))$, the sequence $C_{k}(\omega)$ is uniformly integrable. Finally it follows from Lemma 10 that the sequence $E_{k}(\omega)$ is nondecreasing for each $\omega$. In fact if $\omega$ has no points in $\left[\xi_{v}^{k}, \xi_{v+1}^{k}\right)$ say, and if the points of $\Delta_{k+1}$ in $\left[\xi_{v}^{k}, \xi_{v+1}^{k}\right)$ are $\xi_{v}^{k}=\sigma_{0}<\sigma_{1}<\cdots<\sigma_{m+1}=\xi_{v+1}^{k}$, then $\chi_{\left\{N\left\{\sigma_{j}, \sigma_{j+1}\right)=0\right\}}(\omega)=1$ for every $j=0,1, \ldots, m$ and

$$
P\left(N\left[\xi_{v}^{k}, \xi_{v+1}^{k}\right) \geqq 1 \mid \mathscr{F}_{\xi_{v}^{k}}\right)(\omega) \leqq \sum_{j=0}^{m} P\left(N\left[\sigma_{j}, \sigma_{j+1}\right) \geqq 1 \mid \mathscr{F}_{\xi_{j}}\right)(\omega) .
$$

Further $\int_{\Omega} \sup _{k} E_{k}(\omega) P(d \omega)<\infty$, for otherwise $\int_{\Omega} W(\omega) P(d \omega)$ would be infinite. Thus the sequence $E_{k}(\omega)$ is also uniformly integrable and hence so is $S_{\Delta_{k}}(\omega)$ $=A_{k}(\omega)+C_{k}(\omega)+E_{k}(\omega)$.

Corollary 1. If $t<s$ and if $A \in \mathscr{F}_{t}$ then

$\int_{A} N(\omega,[t, s)) P(d \omega)=\int_{A} W(\omega,[t, s)) P(d \omega)$. I.e., $E\left(N[t, s) \mid \mathscr{F}_{t}\right)=E\left(W[t, s) \mid \mathscr{F}_{t}\right)$. 
Proof. If for each partition $\Delta=\left\{t=\xi_{0}<\xi_{1}<\cdots<\xi_{n+1}=s\right\}$ of $[t, s)$ we construct the sum $\sum_{v=0}^{n} Z\left[\xi_{v}, \xi_{v+1}\right)$ then $W[t, s)=\lim _{\|\Delta\| \rightarrow 0} \sum_{v=0}^{n} Z\left[\xi_{v}, \xi_{v+1}\right)$ in the mean, hence in particular, for $A \in \mathscr{F}_{t}$,

$$
\begin{aligned}
\int_{A} W[t, s) d P & =\lim _{\|\Delta\| \rightarrow 0} \int_{A} \sum_{v=0}^{n} Z\left[\xi_{v}, \xi_{v+1}\right) d P \\
& =\lim _{\|\Delta\| \rightarrow 0} \sum_{\nu=0}^{n} \int_{A} E\left(N\left[\xi_{v}, \xi_{v+1}\right) \mid \mathscr{F}_{\xi_{v}}\right) d P \\
& =\lim _{\|\Delta\| \rightarrow 0} \sum_{\nu=0}^{n} \int_{A} N\left[\xi_{v}, \xi_{v+1}\right) d P=\int_{A} N[t, s) d P .
\end{aligned}
$$

Corollary 2. The process $U(t, \omega)=N(\omega,[0, t))-W(\omega,[0, t)), t>0$, is a martingale.

I am indebted to H. Rost for this observation.

5. The induced past-dependent change of scale. For fixed $\omega, W(\omega,[b, c))$ is a $\sigma$-additive interval function defined for left-closed right-open intervals. (It is finitely additive and "left continuous": given $c$ and $\varepsilon>0$ there is $\delta>0$ such that $W(\omega,[b, c))<\varepsilon$ for all $b$ with $0<c-b<\delta$. This follows from Lemma 9 and the definition of the Burkill integral.) It can therefore be extended to a measure on $\mathscr{B}$, which we denote by $W(\omega, Q)(Q \in \mathscr{B})$. In suggestive notation $W(\omega,[x, x+d x))$ $=E\left(N[x, x+d x) \mid \mathscr{F}_{x}\right)$. Clearly $W(\omega, Q)=W(\omega+x, Q+x)$, hence $W(\omega,[n, n+1))$ $=W(\omega-n,[0,1))$ and by the ergodic theorem $W(\omega,[0, n)) \rightarrow \infty$ a.s. In fact $W(\omega,[0, n)) / n \rightarrow E(W[0,1) \mid \mathscr{J})$ a.s., where $\mathscr{J}$ is the invariant $\sigma$-field of the transformation $\omega \rightarrow \omega-1$. Now $E(W[0,1) \mid \mathscr{J})>0$ a.s. for if

$$
C=\{E(W[0,1) \mid \mathscr{J})=0\} \in \mathscr{J}
$$

then $W[0,1)=0$ on $C$ and, by the invariance of $C, W[0, \infty)=0$ on $C$. The event $W[0, \infty)=0$, however, can easily be seen to have probability zero (this also follows from the argument for the strict monotonicity of (26) below).

Corollary 1 to Theorem 3 easily implies that if $A \in \mathscr{F}_{t}$ and $Q \subseteq[t, \infty)$ then

$$
\int_{A} N(\omega, Q) P(d \omega)=\int_{A} W(\omega, Q) P(d \omega) .
$$

Just as with (3), one can define a measure $\bar{m}$ on $\mathscr{B} \otimes \mathscr{F}$ such that $\bar{m}(Q \times A)$ $=\int_{A} W(\omega, Q) P(d \omega)$ for rectangles $Q \times A$. Proceeding as with $m$, we can define regular "Palm probabilities" $\bar{P}(A \mid x)$ such that

$$
\bar{m}(Q \times A)=\int_{Q} \bar{P}(A \mid x) \alpha d x
$$

and the analogue of (4) is now

$$
\bar{m}(D)=\int_{\Omega} W\left(\omega, D^{\omega}\right) P(d \omega)=\int_{R} \bar{P}\left(D_{x} \mid x\right) \alpha d x \quad(D \in \mathscr{B} \otimes \mathscr{F}) .
$$


Definition. $\mathscr{D}=\left\{D \in \mathscr{B} \otimes \mathscr{F}: D_{t} \in \mathscr{F}_{t}\right.$ for every $\left.t \in R\right\}$. In other words a subset $D$ of $R \times \Omega$ belongs to $\mathscr{D}$ if its characteristic function $\chi_{D}(t, \omega)$ is a $\mathscr{B} \otimes \mathscr{F}$ measurable stochastic process adapted to the $\sigma$-fields $\mathscr{F}_{t}$. We call $\mathscr{D}$ the $\sigma$-field of adapted sets.

THEOREM 4. If $D \in \mathscr{D}$ then $m(D)=\bar{m}(D)$.

Proof. Suppose $D=Q \times A$, where $Q \subseteq[t, \infty)$ and $A \in \mathscr{F}_{t}$. Then by (23) $m(Q \times A)$ $=\bar{m}(Q \times A)$ and it follows from (24) and the fact that $\mu=\alpha \lambda$ that $\int_{Q} P(A \mid x) d x$ $=\int_{Q} \bar{P}(A \mid x) d x$. This implies that if $A \in \mathscr{F}_{t}$ then $P(A \mid x)=\bar{P}(A \mid x)$ for almost all $x \geqq t$. If for each rational number $r$ we choose a countable generating set in $\mathscr{F}_{r}$, $A_{r, 1}, A_{r, 2}, \ldots$, closed under finite intersections, then there is a $\lambda$-null set $M \subseteq R$ such that if $x \notin M$ then $P\left(A_{r, n} \mid x\right)=\bar{P}\left(A_{r, n} \mid x\right)$ for all rationals $r<x$ and all $n$. It follows easily that if $x \notin M$ then $P(A \mid x)=\bar{P}(A \mid x)$ for any $A \in \mathscr{F}_{x}$.

If now $D \in \mathscr{D}$ then $m(D)=\alpha \int_{R} P\left(D_{x} \mid x\right) d x=\alpha \int_{R} \bar{P}\left(D_{x} \mid x\right) d x=\bar{m}(D)$.

LEMMA 11. If $G(x), x \geqq 0$, is a continuous nondecreasing function on $[0, \infty)$ with $G(0)=0$ and if we denote by $v$ the measure induced by $G$ on $[0, \infty)$ then for any Borel set $Q \subseteq[0, \infty)$ we have $\nu\left(G^{-1} Q\right)=\lambda(Q)$.

This is trivial.

The following theorem will be needed below. It is a consequence of $[5$, Theorem 2].

THEOREM 5. Suppose that a point process, which has no multiple points (but is not assumed stationary), satisfies the following condition: For any interval $[s, r]$, $E\left(N[s, r] \mid \mathscr{F}_{s}\right)=\alpha^{\prime}(r-s)$ a.s. (equivalently, if $A \in \mathscr{F}_{t}$ and if $Q$ is an interval contained in $[t, \infty)$, then $\left.\int_{A} N(\omega, Q) P(d \omega)=\alpha^{\prime} \lambda(Q) P(A)\right)$. Then the process is homogeneous Poisson with rate $\alpha^{\prime}$.

The main theorem of this section asserts that if the random transformation $t \Rightarrow W(\omega,[0, t))$ of $[0, \infty)$ onto itself is continuous (i.e. has no jumps) then it transforms the nonnegative points of the given process into a Poisson process in $[0, \infty)$ with rate 1 and independent of $\mathscr{F}_{0}$. A proof of this under the stronger assumption that $P_{0}$ is absolutely continuous relative to $P$ on $\mathscr{F}_{0}$ (which implies as will be shown in $\S 6$ that $W(\omega,[0, t))$ is a.s. absolutely continuous) is given in [5]. The following proof is an adaptation of this.

TheOREM 6. Suppose $W(\omega, \cdot)$ is an atomless measure for P-almost every $\omega$. Let

$$
0 \leqq t_{1}(\omega)<t_{2}(\omega)<t_{3}(\omega)<\cdots
$$

be the nonnegative points of $\omega$ and define the sequence

$$
0 \leqq \tau_{1}(\omega) \leqq \tau_{2}(\omega) \leqq \tau_{3}(\omega) \leqq \cdots
$$

by $\tau_{n}(\omega)=W\left(\omega,\left[0, t_{n}(\omega)\right)\right)$. Under the probability $P$ the sequence (26) is almost surely strictly increasing and, in fact, a homogeneous Poisson process with rate 1 and independent of the $\sigma$-field $\mathscr{F}_{0}$. 
Proof. We can obviously assume that $W(\omega, \cdot)$ is atomless for every $\omega$. Then for every $\omega$ the function $\varphi_{\omega}(t)=Y(t, \omega)=W(\omega,[0, t))$ is continuous and nondecreasing. (For convenience we shall use the symbols $\varphi_{\omega}(t)$ and $Y(t, \omega)$ interchangeably.) Since for fixed $t, W(\omega,[0, t))$ is $\mathscr{F}_{t}$-measurable, the continuity of paths implies that $Y(t, \omega), t \geqq 0$, is measurable relative to $\mathscr{B} \otimes \mathscr{F}$ (in fact progressively measurable relative to the $\sigma$-fields $\mathscr{F}_{t}, t \geqq 0[3$, p. 70]).

We first prove that the sequence (26) is a.s. strictly increasing. Let

$$
\begin{aligned}
K & =\left\{(t, \omega): \text { there is } \varepsilon>0 \text { such that } \varphi_{\omega}(t-\varepsilon)=\varphi_{\omega}(t)\right\} \\
& =\{(t, \omega): \text { there is } \varepsilon>0 \text { such that } W(\omega,[t-\varepsilon, t))=0\} .
\end{aligned}
$$

Note that $K=\bigcup_{n=1}^{\infty}\left\{(t, \omega): \varphi_{\omega}(t-1 / n)=\varphi_{\omega}(t)\right\}$ and hence $K \in \mathscr{B} \otimes \mathscr{F}$, in fact $K \in \mathscr{D}$. Now $\bar{m}(K)=\int_{\Omega} W\left(\omega, K^{\omega}\right) P(d \omega)=0$ since clearly $K^{\omega}=\left(a_{1}(\omega), b_{1}(\omega)\right]$ $\cup\left(a_{2}(\omega), b_{2}(\omega)\right] \cup \cdots$, where $\left[a_{1}(\omega), b_{1}(\omega)\right],\left[a_{2}(\omega), b_{2}(\omega)\right], \ldots$ are the disjoint intervals of constancy of $\varphi_{\omega}$. By Theorem $4, \int_{\Omega} N\left(\omega, K^{\omega}\right) P(d \omega)=m(K)=\bar{m}(K)=0$, therefore $N\left(\omega, K^{\omega}\right)=0$ for $P$-almost all $\omega$, i.e. with probability one $\omega$ has no points in $K^{\omega}$ and this implies that the sequence (26) is strictly increasing because if $W\left(\omega,\left[0, t_{n}(\omega)\right)\right)=W\left(\omega,\left[0, t_{n+1}(\omega)\right)\right)$ then $t_{n+1}(\omega) \in K^{\omega}$. For simplicity we assume this for every $\omega \in \Omega$.

Let now $\Omega^{*}$ be the set of all infinite but locally finite subsets of $[0, \infty)$ and $\mathscr{F} *$ the $\sigma$-field of subsets of $\Omega^{*}$ generated by the random variables $N^{*}\left(\omega^{*}, Q\right)$ $(Q \subseteq[0, \infty))$ (definition obvious). We shall write $\mathscr{F}_{[0, s)}^{*}$ for the $\sigma$-field generated in $\mathscr{F}^{*}$ by the random variables $N^{*}\left(\omega^{*}, Q\right)$ with $Q \subseteq[0, s)$.

One can prove exactly as in [5, Lemma 6] that the mapping $\Omega \ni \omega \Rightarrow S \omega$ $=\left\{\varphi_{\omega}(x): x \in \omega, x \geqq 0\right\} \in \Omega^{*}$ is a Borel mapping of $(\Omega, \mathscr{F})$ into $\left(\Omega^{*}, \mathscr{F}^{*}\right)$. On $\mathscr{F}^{*}$ we introduce the following probabilities. For each $B \in \mathscr{F}_{0}$ with $P(B)>0$ we define

$$
P_{B}^{*}(A)=P\left(S^{-1} A \mid B\right)=P\left(S^{-1} A \cap B\right) / P(B) \quad\left(A \in \mathscr{F}^{*}\right) .
$$

This determines the distribution of the process (26) when restricted to $B$. We shall show that all these distributions are Poisson in $[0, \infty)$ with rate 1 and hence they coincide. This will imply $P_{B}^{*}(A)=P_{\Omega}^{*}(A)$, i.e. $P\left(S^{-1} A \cap B\right)=P\left(S^{-1} A\right) P(B)$ which shows the independence of $\mathscr{F}_{0}$ and the $\sigma$-field generated by (26).

Let $Q=\left[s_{0}, r_{0}\right]$, with $s_{0}>0$, and let $A \in \mathscr{F}_{\left[0, s_{0}\right)}^{*}$. Then denoting by $S_{B}$ the restriction of $S$ to $B$ we have

$$
\begin{aligned}
P(B) \int_{A} N^{*}\left(\omega^{*}, Q\right) P_{B}^{*}\left(d \omega^{*}\right) & =\int_{S_{B}^{-1} A} N(S \omega, Q) P(d \omega) \\
& =\int_{B \cap S^{-1} A} N\left(\omega, \varphi_{\omega}^{-1} Q\right) P(d \omega)=m(D)
\end{aligned}
$$

where $D=\left\{(t, \omega): \omega \in B \cap S^{-1} A, t \in \varphi_{\omega}^{-1} Q\right\}$. Clearly $D \in \mathscr{B} \otimes \mathscr{F}$. It is proved in [5, Lemma 6] that $D_{t} \in \mathscr{F}_{t}$ for every $t$; hence $D \in \mathscr{D}$ and by Theorem 4

$$
m(D)=\bar{m}(D)=\int_{B \cap S^{-1} A} W\left(\omega, \varphi_{\omega}^{-1} Q\right) P(d \omega) .
$$


Now by Lemma $11, W\left(\omega, \varphi_{\omega}^{-1} Q\right)=\lambda(Q)$ for any $\omega$, therefore

$$
m(D)=\lambda(Q) P\left(B \cap S^{-1} A\right),
$$

i.e.

$$
\int_{A} N^{*}\left(\omega^{*}, Q\right) P_{B}^{*}\left(d \omega^{*}\right)=\lambda(Q) P_{B}^{*}(A)
$$

This implies by Theorem 5 (as it applies to $[0, \infty)$ ) that $P_{B}^{*}$ defines a Poisson process with rate 1 . The proof is complete.

Theorem 6 is not surprising in view of the remark following (19), which could presumably provide an alternative approach.

If $\Delta=\left\{0=\xi_{0}<\xi_{1}<\cdots\right\}$ is a partition of $[0, \infty)$ we define a random transformation $t \Rightarrow T^{\Delta}(t, \omega)$ of $[0, \infty)$ onto itself by setting first

$$
T^{\Delta}\left(\xi_{n}, \omega\right)=\sum_{v=0}^{n-1} Z\left(\omega,\left[\xi_{v}, \xi_{v+1}\right)\right), \quad n=1,2, \ldots,
$$

and then interpolating $T^{\Delta}(t, \omega)$ linearly for the other $t$ 's. Note that $T^{\Delta}(t, \omega)$ is the time at which we shall pass $t$ if we adopt the following pattern of motion, starting at 0 : At each $\xi_{v}$ we calculate the "expected number of points in $\left[\xi_{v}, \xi_{v+1}\right)$ given $\mathscr{F}_{\xi_{v}}$ " and move to $\xi_{v+1}$ with constant velocity $\left(E\left(N\left[\xi_{v}, \xi_{v+1}\right) \mid \mathscr{F}_{\xi_{v}}\right)\right)^{-1}\left(\xi_{v+1}-\xi_{v}\right)$, i.e. so as to meet these expected points in $\left[\xi_{v}, \xi_{v+1}\right)$ at an average rate of one per time unit. From Theorem 1 and Theorem 6 we obtain

THEOREM 7. Suppose $W$ is a.s. atomless, let (25) be the sequence of nonnegative elements of $\omega$ and for each partition $\Delta$ of $[0, \infty)$ define the sequence

$$
0 \leqq \tau_{1}^{\Delta}(\omega) \leqq \tau_{2}^{\Delta}(\omega) \leqq \cdots
$$

by $\tau_{n}^{\Delta}(\omega)=T^{\Delta}\left(t_{n}(\omega), \omega\right)$. When the mesh of $\Delta$ in each finite interval $[0, r]$ goes to zero, the process (28) converges $P$-almost surely to a Poisson process with rate 1 and independent of $\mathscr{F}_{0}$.

This almost sure convergence can be taken to mean $\tau_{n}^{\Delta}(\omega) \rightarrow \tau_{n}(\omega)$ a.s. for each $n$ $\left(\tau_{n}(\omega)\right.$ as in (26)) but can also be defined in terms of the natural topology in $\Omega^{*}$ indicated in $\S 2$. A weaker version of Theorem 7 is given in [5].

In view of Theorem 7 it is natural to interpret Theorem 6 as follows (see [5]): Suppose an observer, who at each instant is able to see only the past, travels on $[0, \infty)$, regulating his motion (on the basis of up-to-the-moment information on the past) so as to meet expected future points at an instantaneous rate of one per time unit. Then he will meet the actual points of the given process at the times of a Poisson process with rate 1 and independent of $\mathscr{F}_{0}$.

The next theorem gives a criterion for the atomlessness of $W$.

THEOREM 8. $W$ is a.s. atomless if and only if for $P_{0}\left(\eta_{-1} \in d x_{1}, \eta_{-2} \in d x_{2}, \ldots\right)-$ almost all $\left(x_{1}, x_{2}, \ldots\right)$ the conditional distribution

$$
F(x)=P_{0}\left(\eta_{0}<x \mid \eta_{-1}=x_{1}, \eta_{-2}=x_{2}, \ldots\right)
$$

is continuous. 
This follows from the identification of $W$ in the proof of Theorem 1 (see (15) and (19)) and Lemma 2. In fact if the conditional distributions of $\eta_{0}$ given $\eta_{-1}=x_{1}$, $\eta_{-2}=x_{2}, \ldots$ or given $\eta_{-1}=x_{2}, \eta_{-2}=x_{3}, \ldots$ are both continuous, then (15) is zero and (19) is a right-continuous function of $c_{0}$. Conversely, if for some $\omega_{0}, W$ has an atom, say $W\left(\omega_{0},\{x\}\right)>0$, then from the proof of Theorem 1 it is easily seen that an expression of the form (15) is $>0$.

6. A condition of absolute continuity. In the present section we shall be concerned with the case in which $P_{0}$ is absolutely continuous relative to $P$ on the $\sigma$ field $\mathscr{F}_{0}$. (Note that $\Omega_{0}$, which carries $P_{0}$, is not in $\mathscr{F}_{0}$.) Below we shall use the symbol « to denote absolute continuity of one measure relative to another.

Suppose $P_{0} \ll P$ on $\mathscr{F}_{0}$ and let $X(\omega)$ be the corresponding $\mathscr{F}_{0}$-measurable RadonNikodym derivative:

$$
P_{0}(A)=\int_{A} X(\omega) P(d \omega) \quad\left(A \in \mathscr{F}_{0}\right)
$$

Observe that $E X=1$. The process $X(t, \omega)=X(\omega-t)(t \in R, \omega \in \Omega)$ is stationary under $P$, measurable and adapted to the $\sigma$-fields $\mathscr{F}_{t},-\infty<t<\infty$, i.e. for each fixed $t$, the random variable $X(t, \cdot)$ is $\mathscr{F}_{t}$-measurable. Recall that a measurable stationary $L_{1}$-process is mean continuous:

$$
\lim _{t \rightarrow t_{0}} E\left|X(t)-X\left(t_{0}\right)\right|=0 .
$$

Note also that

$$
P(A \mid t)=\int_{A} X(t, \omega) P(d \omega) \quad\left(A \in \mathscr{F}_{t}\right) .
$$

THEOREM 9. The condition $P_{0} \ll P$ on $\mathscr{F}_{0}$ is necessary and sufficient for mean convergence in Theorem 2 (or equivalently in $\lim _{\varepsilon \downarrow 0} \varepsilon^{-1} E\left(N[0, \varepsilon) \mid \mathscr{F}_{0}\right)$ ). In this case $Y(\omega)=\alpha X(\omega)$ a.s.

Proof. Sufficiency of the condition " $P_{0} \ll P$ on $\mathscr{F}_{0}$ " is proved in [5, Theorem 6].

For the necessity suppose $\lim _{\varepsilon \downarrow 0} \varepsilon^{-1} E\left(N[0, \varepsilon) \mid \mathscr{F}_{0}\right)=Y$ exists in the mean. Given any interval $[b, c) \subseteq(-\infty, 0)$ and any nonnegative integer $j$ let $V(\omega)$ $=\chi_{\{N[b, c)=j\}}(\omega)$. Then

$$
\begin{aligned}
\int_{\Omega} V Y d P & =\lim _{\varepsilon \downarrow 0} \varepsilon^{-1} \int_{\Omega} V \cdot E\left(N[0, \varepsilon) \mid \mathscr{F}_{0}\right) d P=\lim _{\varepsilon \downarrow 0} \varepsilon^{-1} \int_{\Omega} V \cdot N[0, \varepsilon) d P \\
& =\lim _{\varepsilon \downarrow 0} \varepsilon^{-1} \int_{0}^{\varepsilon} \int_{\Omega} V(\omega) P(d \omega \mid x) \alpha d x \text { by (3) } \\
& =\lim _{\varepsilon \downarrow 0} \varepsilon^{-1} \int_{0}^{\varepsilon} \int_{\Omega} V(\omega+x) P_{0}(d \omega) \alpha d x .
\end{aligned}
$$

Now $V(\omega+x) \rightarrow V(\omega)$ when $x \downarrow 0$, hence $\int_{\Omega} V(\omega+x) P_{0}(d \omega) \rightarrow \int_{\Omega} V(\omega) P_{0}(d \omega)$ and therefore $\int_{\Omega} V Y d P=\alpha \int_{\Omega} V d P_{0}$. Since this is also true if $V$ is a finite product of characteristic functions of the above type, we conclude $Y d P=\alpha d P_{0}$. 
THEOREM 10. If $P_{0} \ll P$ on $\mathscr{F}_{0}$, then for $P$-almost all $\omega \in \Omega$,

$$
W(\omega, Q)=\alpha \int_{Q} X(t, \omega) d t \text { for all } Q \in \mathscr{B} \text {. }
$$

Conversely if for P-almost all $\omega \in \Omega$ the measure $W(\omega, \cdot)$ is absolutely continuous relative to $\lambda$ then $P_{0} \ll P$ on $\mathscr{F}_{0}$.

Proof. See [5, Theorem 6] for the first half of the theorem. To prove the second half suppose $W$ is a.s. absolutely continuous. If we define

$$
X(t, \omega)=\alpha^{-1} \cdot \lim _{\varepsilon \downarrow 0} \varepsilon^{-1} W(\omega,[t-\varepsilon, t))
$$

then $X(t, \omega)$ is measurable, stationary and adapted to $\mathscr{F}_{t}, t \in R$, and $W(\omega,[b, c))$ $=\alpha \int_{b}^{c} X(t, \omega) d t$ a.s. Corollary 1 of Theorem 3 now implies

$$
E\left(N[x, y) \mid \mathscr{F}_{x}\right)=E\left(\alpha \int_{x}^{y} X(t) d t \mid \mathscr{F}_{x}\right)
$$

and the proof of [5, Lemma 7] shows that this entails the existence of $\lim _{\varepsilon \downarrow 0} \varepsilon^{-1} E\left(N[0, \varepsilon) \mid \mathscr{F}_{0}\right)$ in the mean. The result follows from Theorem 9.

The second half of this theorem will also be obtained as a consequence of Theorem 13 below. Comparing with (30) we see that, for fixed $t, X(t, \cdot)$ is the Radon-Nikodym derivative of $P(\cdot \mid t)$ relative to $P(\cdot)$, while for fixed $\omega, \alpha X(\cdot, \omega)$ is the Radon-Nikodym derivative of $W(\omega, \cdot)$ relative to $\lambda(\cdot)$.

Proposition 2 in [5] states that the following conditions are equivalent.

(a) $P_{0} \ll P$ on $\mathscr{F}_{0}$.

(b) $\lim _{t \downarrow 0}\left(\sup _{A \in \mathscr{F}_{0}}|P(A \mid t)-P(A \mid 0)|\right)=0$.

(c) For each $A \in \mathscr{F}_{0}, \lim _{t \downarrow 0} P(A \mid t)=P(A \mid 0)$.

The proof is almost immediate and in fact conditions (b) and (c) shed little light on (a). The following theorem gives a more useful criterion. Recall that $\mathscr{F}_{0}$ is generated by the random variables $\theta(\omega), \zeta_{-1}(\omega), \zeta_{-2}(\omega), \ldots$

THEOREM 11. The following conditions are equivalent.

(i) $P_{0} \ll P$ on $\mathscr{F}_{0}$.

(ii) $P_{0}\left(\eta_{0} \in d x, \eta_{-1} \in d x_{1}, \eta_{-2} \in d x_{2}, \ldots\right) \ll d x \cdot P_{0}\left(\eta_{-1} \in d x_{1}, \eta_{-2} \in d x_{2}, \ldots\right)$.

(iii) $P_{0}\left(\eta_{0} \in d x, \eta_{-1} \in d x_{1}, \eta_{-2} \in d x_{2}, \ldots\right) \ll P_{0}\left(\eta_{0} \in d x\right) \cdot P_{0}\left(\eta_{-1} \in d x_{1}, \eta_{-2} \in d x_{2}\right.$, ...) and $P_{0}\left(\eta_{0} \in d x\right) \ll d x$.

(iv) There is a $\sigma$-finite measure $\pi\left(d x_{1}, d x_{2}, \ldots\right)$ on $R_{+}^{\infty}$ such that

$$
P_{0}\left(\eta_{0} \in d x, \eta_{-1} \in d x_{1}, \eta_{-2} \in d x_{2}, \ldots\right) \ll d x \cdot \pi\left(d x_{1}, d x_{2}, \ldots\right) .
$$

Proof. We shall prove (i) $\Rightarrow$ (ii) $\Rightarrow$ (iv) $\Rightarrow$ (i) and (ii) $\Leftrightarrow$ (iii).

Suppose (i) is true, i.e.

$$
P_{0}\left(\theta \in d x, \zeta_{-1} \in d x_{1}, \zeta_{-2} \in d x_{2}, \ldots\right) \ll P\left(\theta \in d x, \zeta_{-1} \in d x_{1}, \zeta_{-2} \in d x_{2}, \ldots\right) .
$$

From (5) and Lemma 3 we have

$$
P\left(\theta \in d x, \zeta_{-1} \in d x_{1}, \zeta_{-2} \in d x_{2}, \ldots\right) \ll d x \cdot P_{0}\left(\zeta_{0} \in d x_{1}, \zeta_{-1} \in d x_{2}, \ldots\right)
$$


hence (i) implies

$$
P_{0}\left(\theta \in d x, \zeta_{-1} \in d x_{1}, \zeta_{-2} \in d x_{2}, \ldots\right) \ll d x \cdot P_{0}\left(\zeta_{0} \in d x_{1}, \zeta_{-1} \in d x_{2}, \ldots\right) .
$$

Since $P_{0}$ is carried by $\Omega_{0}$ this means

$$
P_{0}\left(\eta_{-1} \in d x, \eta_{-2} \in d x_{1}, \eta_{-3} \in d x_{2}, \ldots\right) \ll d x \cdot P_{0}\left(\eta_{-1} \in d x_{1}, \eta_{-2} \in d x_{2}, \ldots\right)
$$

which is (ii) because $P_{0}$ is shift invariant.

That (ii) implies (iv) is trivial. We now prove (iv) $\Rightarrow$ (i). Let $f(\omega)$ be an $\mathscr{F}_{0^{-}}$ measurable function such that $0 \leqq f \leqq 1$ and $\int_{\Omega} f(\omega) P(d \omega)=0$. We shall prove that $\int_{\Omega} f(\omega) P_{0}(d \omega)=0$. Suppose $f(\omega)=g\left(\theta(\omega), \zeta_{-1}(\omega), \zeta_{-2}(\omega), \ldots\right)$, then by Lemma 1

$$
\begin{aligned}
0 & =\int_{\Omega} g\left(\theta(\omega), \zeta_{-1}(\omega), \zeta_{-2}(\omega), \ldots\right) P(d \omega) \\
& =\alpha \int_{0}^{\infty} \int_{\left\{\eta_{0} \geqq y\right\}} g\left(y, \eta_{-1}(\omega), \eta_{-2}(\omega), \ldots\right) P_{0}(d \omega) d y \\
& =\alpha \int_{0}^{\infty} \int_{C_{y}} g\left(y, x_{1}, x_{2}, \ldots\right) P_{0}\left(\eta_{0} \in d x, \eta_{-1} \in d x_{1}, \ldots\right) d y
\end{aligned}
$$

where $C_{y}=\left\{\left(x, x_{1}, x_{2}, \ldots\right): x \geqq y\right\}=[y ; \infty) \times R_{+} \times R_{+} \times \ldots$ If $\varphi\left(x, x_{1}, x_{2}, \ldots\right)$ is the Radon-Nikodym derivative of $P_{0}\left(\eta_{0} \in d x, \eta_{-1} \in d x_{1}, \ldots\right)$ relative to

$$
d x \cdot \pi\left(d x_{1}, d x_{2}, \ldots\right)
$$

then

$$
\begin{aligned}
0 & =\alpha \int_{0}^{\infty} \int_{R_{+}^{\infty}} \int_{y}^{\infty} g\left(y, x_{1}, x_{2}, \ldots\right) \varphi\left(x, x_{1}, x_{2}, \ldots\right) d x \pi\left(d x_{1}, d x_{2}, \ldots\right) d y \\
& =\alpha \int_{0}^{\infty} \int_{R_{+}^{\infty}} \int_{0}^{\infty} g\left(y, x_{1}, \ldots\right) \varphi\left(y+x, x_{1}, \ldots\right) d x \pi\left(d x_{1}, d x_{2}, \ldots\right) d y \\
& =\alpha \int_{R_{+}^{\infty}} \int_{0}^{\infty} \int_{0}^{\infty} g\left(y, x_{1}, \ldots\right) \varphi\left(y+x, x_{1}, \ldots\right) d y d x \pi\left(d x_{1}, d x_{2}, \ldots\right)
\end{aligned}
$$

By the Fubini theorem there is a $\pi$-null set $K \subseteq R_{+}^{\infty}$ such that if $\left(x_{1}, x_{2}, \ldots\right) \notin K$ then for almost all $x \geqq 0$

$$
\int_{0}^{\infty} g\left(y, x_{1}, \ldots\right) \varphi\left(y+x, x_{1}, \ldots\right) d y=0 .
$$

This implies $\int_{0}^{\infty} g\left(y, x_{1}, \ldots\right) \varphi\left(y, x_{1}, \ldots\right) d y=0$, hence

$$
\begin{array}{r}
\int_{R_{+}^{\infty}} \int_{0}^{\infty} g\left(y, x_{1}, \ldots\right) \varphi\left(y, x_{1}, \ldots\right) d y \pi\left(d x_{1}, d x_{2}, \ldots\right)=0, \\
\int_{R_{+}^{\infty}} g\left(y, x_{1}, \ldots\right) P_{0}\left(\eta_{0} \in d y, \eta_{-1} \in d x_{1}, \ldots\right)=0
\end{array}
$$

from which we easily obtain $\int_{\Omega_{0}} f(\omega) P_{0}(d \omega)=0$. 
The implication (ii) $\Rightarrow$ (iii) is almost immediate: If $B \subseteq R_{+}^{\infty}$ is such that $\int_{B} P_{0}\left(\eta_{0} \in d x\right) P_{0}\left(\eta_{-1} \in d x_{1}, \ldots\right)=0$ then there is a set $M \subseteq R_{+}$such that $P_{0}\left(\eta_{0} \in M\right)$ $=0$ and, for all $x \in M^{c}, P_{0}\left(\left(\eta_{-1}, \eta_{-2}, \ldots\right) \in B_{x}\right)=0$ where

$$
B_{x}=\left\{\left(x_{1}, x_{2}, \ldots\right):\left(x, x_{1}, x_{2}, \ldots\right) \in B\right\} .
$$

From this,

$$
\int_{M^{c}} P_{0}\left(\left(\eta_{-1}, \eta_{-2}, \ldots\right) \in B_{x}\right) d x=0
$$

and by hypothesis (ii) this implies $P_{0}\left(\eta_{0} \in M^{c}\right.$ and $\left.\left(\eta_{0}, \eta_{-1}, \ldots\right) \in B\right)=0$ and hence $P_{0}\left(\left(\eta_{0}, \eta_{-1}, \ldots\right) \in B\right) \leqq P_{0}\left(\eta_{0} \in M\right)+P_{0}\left(\eta_{0} \in M^{c}\right.$ and $\left.\left(\eta_{0}, \eta_{-1}, \ldots\right) \in B\right)=0$. Finally, (iii) $\Rightarrow$ (ii) trivially and the proof is complete.

THEOREM 12. If $P_{0} \ll P$ on $\mathscr{F}_{-\varepsilon}$ for every $\varepsilon>0$ then $P_{0} \ll P$ on $\mathscr{F}_{0}$.

Proof. By Theorem 11 it is sufficient to prove

$$
P_{0}\left(\eta_{0} \in d x, \eta_{-1} \in d x_{1}, \ldots\right) \ll d x \cdot P_{0}\left(\eta_{-1} \in d x_{1}, \ldots\right) .
$$

Consider a Borel function $g\left(x, x_{1}, x_{2}, \ldots\right)$ on $R_{+}^{\infty}$ with $0 \leqq g \leqq 1$ and suppose that $\int_{\Omega} g\left(\eta_{0}(\omega), \eta_{-1}(\omega), \ldots\right) P_{0}(d \omega)>0$ or equivalently $\int_{\Omega} g\left(\eta_{-1}(\omega), \eta_{-2}(\omega), \ldots\right) P_{0}(d \omega)$ $>0$. Then, for some $\varepsilon>0$,

$$
\int_{\left\{\eta_{-1}>\varepsilon\right\}} g\left(\eta_{-1}(\omega), \eta_{-2}(\omega), \ldots\right) P_{0}(d \omega)>0 .
$$

Let us define $k_{-1}(\omega ;-\varepsilon)=-t_{0}(\omega ;-\varepsilon), k_{i}(\omega ;-\varepsilon)=t_{i+2}(\omega ;-\varepsilon)-t_{i+1}(\omega ;-\varepsilon)$, $i=-2,-3, \ldots$ On the set $\left\{\eta_{-1}>\varepsilon\right\}$ we have $\eta_{i}(\omega)=k_{i}(\omega ;-\varepsilon), i=-1,-2, \ldots$ Hence by (32)

$$
\int_{\Omega} g\left(k_{-1}(\omega ;-\varepsilon), k_{-2}(\omega ;-\varepsilon), \ldots\right) P_{0}(d \omega)>0 .
$$

Now $g\left(k_{-1}(\omega ;-\varepsilon), k_{-2}(\omega ;-\varepsilon), \ldots\right)$ is $\mathscr{F}_{-\varepsilon}$-measurable and by hypothesis we must have

$$
\int_{\Omega} g\left(k_{-1}(\omega ;-\varepsilon), k_{-2}(\omega ;-\varepsilon), \ldots\right) P(d \omega)>0 .
$$

The stationariness of $P$ implies

$$
\int_{\Omega} g\left(k_{-1}(\omega-\varepsilon ;-\varepsilon), k_{-2}(\omega-\varepsilon ;-\varepsilon), \ldots\right) P(d \omega)>0 .
$$

Since $k_{-1}(\omega-\varepsilon ;-\varepsilon)=-t_{0}(\omega-\varepsilon ;-\varepsilon)=\varepsilon+\theta(\omega)$ while $k_{i}(\omega-\varepsilon ;-\varepsilon)=\zeta_{i+1}(\omega)$ for $i=-2,-3, \ldots$ we obtain

$$
\int_{\Omega} g\left(\varepsilon+\theta(\omega), \zeta_{-1}(\omega), \zeta_{-2}(\omega), \ldots\right) P(d \omega)>0 .
$$


By Lemma 1

$$
\begin{array}{r}
\int_{\Omega_{0}} \int_{0}^{\infty} g\left(\varepsilon+x, \eta_{-1}(\omega), \eta_{-2}(\omega), \ldots\right) d x P_{0}(d \omega)>0 \\
\int_{\Omega_{0}} \int_{\varepsilon}^{\infty} g\left(x, \eta_{-1}(\omega), \eta_{-2}(\omega), \ldots\right) d x P_{0}(d \omega)>0
\end{array}
$$

and (31) follows.

Theorem 12 enables us to improve Theorem 7 of [5].

THEOREM 13. Given a stationary point process in $(-\infty, \infty)$, suppose there exists a measurable nonnegative stochastic process $X(t, \omega), t \geqq 0$, on $\Omega$ with the following property: If for each $\omega$ we set $\tau_{n}(\omega)=\int_{0}^{t_{n}(\omega)} X(x, \omega) d x$ (where $t_{n}(\omega)$ is as in (25)) then the process

$$
\tau_{1}(\omega) \leqq \tau_{2}(\omega) \leqq \cdots
$$

is (i) a.s. strictly increasing, (ii) independent of $\mathscr{F}_{0}$ and (iii) first order stationary, i.e. there is an $\alpha^{\prime}>0$ such that the expected number of members of (33) in any Borel set $Q \subseteq[0, \infty)$ is $\alpha^{\prime} \cdot \lambda(Q)$. Then $P_{0} \ll P$ on $\mathscr{F}_{0}$.

This is proved in [5, Theorem 7], under the additional assumption that $X(t)$, $t \geqq 0$, is stationary. We can now dispense with this assumption. We prove exactly as in [5] that $P_{0} \ll P$ on $\mathscr{F}_{-\varepsilon}$ for every $\varepsilon>0$ and then invoke Theorem 12 to deduce that $P_{0} \ll P$ on $\mathscr{F}_{0}$. A corollary of Theorems 6 and 13 is the second half of Theorem 10. Note that the latter can also be deduced from the weaker version of Theorem 13 given in [5], since if $W(\omega,[0, t))$ is absolutely continuous then its derivative $X(t, \omega)$ is measurable and stationary.

The last theorem is an analogue of Theorem 8.

THEOREM 14. $W$ is a.s. absolutely continuous if and only if for

$$
P_{0}\left(\eta_{-1} \in d x_{1}, \eta_{-2} \in d x_{2}, \ldots\right) \text {-almost all }\left(x_{1}, x_{2}, \ldots\right) \in R_{+}^{\infty}
$$

the conditional distribution $F(x)=P_{0}\left(\eta_{0}<x \mid \eta_{-1}=x_{1}, \eta_{-2}=x_{2}, \ldots\right)$ is absolutely continuous.

This is simply another way of stating condition (ii) of Theorem 11. It is clear that the latter is implied by the condition of the present theorem (Fubini!). We now prove the converse: Suppose (ii) (Theorem 11) is true and let $Q_{1}, Q_{2}, \ldots$ be an enumeration of all intervals in $(0, \infty)$ with rational endpoints. If $\varphi\left(x, x_{1}, x_{2}, \ldots\right)$ is the Radon-Nikodym derivative whose existence is implied by (ii), then for any Borel set $S \subseteq R_{+}^{\infty}$

$$
\begin{aligned}
\int_{S P} P_{0}\left(\eta_{0} \in Q_{i} \mid \eta_{-1}=x_{1}, \eta_{-2}=x_{2}, \ldots\right) P_{0}\left(\eta_{-1} \in d x_{1}, \eta_{-2} \in d x_{2}, \ldots\right) \\
=P_{0}\left(\eta_{0} \in Q_{i} \text { and }\left(\eta_{-1}, \eta_{-2}, \ldots\right) \in S\right) \\
=\int_{S} \int_{Q_{1}} \varphi\left(x, x_{1}, x_{2}, \ldots\right) d x P_{0}\left(\eta_{-1} \in d x_{1}, \eta_{-2} \in d x_{2}, \ldots\right)
\end{aligned}
$$


Hence there exists a $P_{0}\left(\eta_{-1} \in d x_{1}, \ldots\right)$-null set $M_{i} \subseteq R_{+}^{\infty}$ such that

$$
P_{0}\left(\eta_{0} \in Q_{i} \mid \eta_{-1}=x_{1}, \ldots\right)=\int_{Q_{i}} \varphi\left(x, x_{1}, \ldots\right) d x
$$

for all $\left(x_{1}, x_{2}, \ldots\right) \notin M_{i}$. Clearly if $\left(x_{1}, x_{2}, \ldots\right) \notin \bigcup_{i} M_{i}$ then

$$
P_{0}\left(\eta_{0} \in Q \mid \eta_{-1}=x_{1}, \ldots\right)=\int_{Q} \varphi\left(x, x_{1}, \ldots\right) d x
$$

for any Borel set $Q \subseteq R_{+}$.

This theorem follows also (just as Theorem 8) from the identification of $W$ given in the proof of Theorem 1. One can therefore base a proof of Theorem 11 on Theorems 10 and 14.

\section{REFERENCES}

1. N. Bourbaki, Livre VI: Intégration, Chap. 4, Actualités Sci. Indust., no. 1175, Hermann, Paris, 1952. MR 14, 960.

2. W. Fieger, Die Anzahl der $\gamma$-Niveau-Kreuzungspunkte von stochastischen Prozessen, $\mathrm{Z}$. Wahrscheinlichkeitstheorie und Verw. Gebiete 18 (1971), 227-260.

3. P. A. Meyer, Probability and potentials, Publ. Inst. Math. Univ. Strasbourg, no. 14, Actualités Sci. Indust., no. 1318, Hermann, Paris, 1966; English transl., Blaisdell, Waltham, Mass., 1966. MR 34 \#5118; MR 34 \#5119.

4. K. Murali Rao, On decomposition theorems of Meyer, Math. Scand. 24 (1969), 66-78.

5. F. Papangelou, On the Palm probabilities of processes of points and processes of lines, Memorial Volume to R. Davidson (edited by D. G. Kendall) (to appear).

6. - Summary of some results on point and line processes, Proc. IBM Conference on Stochastic Point Processes (held August 1971).

7. F. Riesz and B. Sz.-Nagy, Leçons d'analyse fonctionnelle, 2nd ed., Akad. Kiadó, Budapest, 1953; English transl., Ungar, New York, 1955. MR 17, 175.

8. C. Ryll-Nardzewski, Remarks on processes of calls, Proc. Fourth Berkeley Sympos. Math. Statist. and Prob., vol. 2, Univ. California Press, Berkeley, Calif., 1961, pp. 455-465. MR 25 \#3575.

9. F. Zitek, The theory of ordinary streams, Czechoslovak Math. J. 8 (83) (1958), 448-459; English transl., Selected Transl. Math. Statist. and Prob., vol. 2, Amer. Math. Soc., Providence, R. I., 1962, pp. 241-251. MR 21 \#376; MR 27 \#814.

Department of Mathematics, Ohio State University, Columbus, Ohio 43210

Current address: Institut für mathematische Statistik, 34 Göttingen, Bürgerstrasse 32, W. Germany 